“@ 2018 IEEE. Personal use of this material is permitted. Permission from IEEE must be obtained for all other uses, in any current or future media, including reprinting/republishing this material for advertising or promotional purposes, creating new collective works, for resale or redistribution to servers or lists, or reuse of any copyrighted component of this work in other works." 


\title{
A Cross-domain Recommender System with Kernel-induced Knowledge Transfer for Overlapping Entities
}

\author{
Qian Zhang, Member, IEEE, Jie Lu, Fellow, IEEE, Dianshuang Wu, Member, IEEE and Guangquan Zhang
}

\begin{abstract}
The aim of recommender systems is to automatically identify user preferences within collected data, then use those preferences to make recommendations that help with decisions. However, recommender systems, particularly those based on collaborative filtering, suffer from data sparsity problem. This issue is particularly prevalent in newly-launched systems that have not yet had enough time to amass sufficient data. As a solution, crossdomain recommender systems transfer knowledge from a source domain with relatively rich data to assist recommendations in the target domain. These systems usually assume that the entities either fully overlap or do not overlap at all. However, in practice, it is more common for the entities in the two domains to partially overlap. Moreover, overlapping entities may have different expressions in each domain. In cross-domain recommender systems, neglecting these two issues reduces prediction accuracy in the target domain. To fully exploit partially-overlapping entities and improve the accuracy of predictions, this paper presents a crossdomain recommender system based on kernel-induced knowledge transfer, called KerKT. Domain adaptation is used to adjust the feature spaces of overlapping entities, while diffusion kernel completion is used to correlate the non-overlapping entities between the two domains. With this approach, knowledge is effectively transferred through the overlapping entities, thus alleviating data sparsity issues. Experiments conducted on four datasets, each with three sparsity ratios, show that KerKT outperforms six benchmarks and increases the accuracy of recommendations in the target domain. KerKT is compared with six methods and has $1.13 \%-20 \%$ better prediction accuracy in terms of MAE and RMSE respectively. Additionally, the results indicate that transferring knowledge from the source domain to the target domain is both possible and beneficial with even small overlaps.
\end{abstract}

Index Terms-recommender systems, cross-domain recommender systems, knowledge transfer, collaborative filtering

\section{INTRODUCTION}

$\mathbf{R}$ ECOMMENDER systems have developed quickly with the explosion of Web 2.0 technologies [1], and are now in wide use. The aim of recommender systems is to provide users with items, such as products or services, that match their preferences. Generally, recommendation techniques are roughly divided into three categories based on the underlying data used to make the recommendation: collaborative filtering-based [2], content-based [3] and knowledge-based recommendation [4]. Collaborative filtering generates recommendations to one user

Q. Zhang, J. Lu, D. Wu and G. Zhang are with the Decision Systems and e-Service Intelligence Laboratory, Center for Artificial Intelligence, Faculty of Engineering and Information Technology, University of Technology Sydney, Sydney, NSW 2007, Australia (e-mail: qian.zhang-1@uts.edu.au; jie.lu@uts.edu.au; dianshuang.wu@uts.edu.au; guangquan.zhang@uts.edu.au).(Corresponding author: Jie Lu.) from the historical records of other users with similar behavior [5]. This approach has advantages when historical data for users and items are actually available, such as ratings or browsing data. Originally designed as basic memory-based methods, collaborative filtering has evolved into model-based methods that commonly involve machine learning techniques, such as matrix factorization [6], probabilistic models [7] and deep neural networks [8], [9], [10]. Matrix factorization is, perhaps, the most widely used and has been incorporated into many commercial recommender systems [11]. However, observing the interactions between users and items has limitations in practice and, among them, data sparsity is a common problem [12]- a problem that is particularly severe and challenging in newly-launched recommender systems. Data sparsity greatly impairs a recommender system's ability to produce accurate recommendation results, which leads to a poor experience for users [13].

To overcome data sparsity issues, some recommender systems based on collaborative filtering are beginning to incorporate transfer learning. Transfer learning extracts shared knowledge from a domain with comparatively denser data [14] and uses that knowledge to improve recommendations in the target domain. In newly-launched recommender systems, this technique can significantly improve performance [15]. Systems that use transfer learning techniques are known as crossdomain recommender systems. These systems are specifically designed to provide recommendations in a target domain using information extracted from a source domain. However, the most crucial concern in cross-domain recommender systems is how to extract common knowledge that can be shared between the two domains.

The methods for knowledge extraction and transfer are different depending on whether and how the entities in each domain overlap. Existing cross-domain recommender systems usually assume that either none of the entities are common to both domains, or they all are with full one-to-one mapping. Non-overlapping methods tend to extract shared knowledge based on collective group-level user behavior. Although many of these methods have been designed to suit specific situations, they cannot integrate knowledge from an overlapping entity once new information becomes available. In fully-overlapping methods, the original source and target rating matrixes are collectively factorized, then the entities' features are extracted. Constraints on each entity ensure these features are exactly the same in the source and target domains so they can act as a bridge for knowledge transfer. However, practical situations 

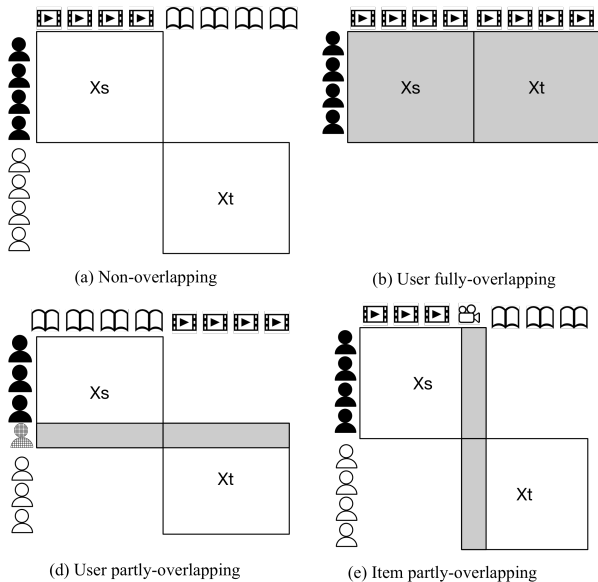

Fig. 1. Different scenarios of overlapping entities.

rarely satisfy the "all" or "none" overlap assumption; rather, they fall somewhere in between as shown in Figure 1. In fully-overlapping methods, information about the overlapping entities is used to establish constraints between two domains. These constraints usually relate to the entities' features. However, even with the same user, there may be small differences in item rating patterns between two different domains, and this is called domain divergence. If the entity feature constraints are not handled delicately, knowledge transfer will suffer and reduce the accuracy of the predictions.

Hence, cross-domain recommendation systems present the following challenges: 1) Feature inconsistency caused by data sparsity. Typically, there are no explicit features, only extracted latent features. And the observed sparse ratings do not fully represent a user's preferences, so features extracted from the same user in two different domains will be inconsistent. Thus, constructing an appropriate feature space is very challenging. 2) Feature inconsistency caused by domain heterogeneity. Extracted latent features from an overlapping entity can be aligned through domain adaptation techniques. But extracted latent features from non-overlapping entities lack a direct correlation and their features are heterogeneous. 3) Partially overlapping entities. The number of overlapping entities can account for a very small part of the total number of entities in the target domain. Whether transferring knowledge through a small number of overlapping entities is effective, and what the shared constraints should be, remain unsolved problems.

In this paper, we propose a cross-domain recommender system with kernel-induced knowledge transfer (KerKT) as a knowledge transfer method to improve recommendation performance with partially overlapping entities. We first factorize the rating matrixes separately to construct the user and item feature matrixes. To avoid divergence in the feature space caused by data sparsity, we propose a domain adaptation method to adjust the feature spaces through the overlapping entities. Then, we use a diffusion kernel to construct a full and complete entity similarity matrix, so the similarity measures can be used in heterogeneous settings. Finally, we use a more flexible constraint to jointly factorize the source and target rating matrixes. The main contributions of this study are as follows:

1) A domain adaptation method that aligns the feature spaces of overlapping entities. The method matches the features of each overlapping entity acquired from two different domains. The overlapping entities are projected onto the same subspace to ensure consistency between the representations. Feature divergence caused by data sparsity is eliminated.

2) A kernel-induced completion method for computing entity similarities in heterogeneous situations. Feature divergence caused by domain heterogeneity is eliminated and domain connection is reinforced. This means the similarities between entities can be determined through a modest amount of overlapping entity data.

3) A new matrix factorization method with constraints that integrates the intra-domain and inter-domain entity correlations acquired from overlapping entities. The two rating matrixes are collectively factorized sharing interdomain knowledge while retaining their own domainspecific characteristics. The constraints are more flexible than previous methods and ensure that more useful knowledge is transferred to the target domain.

4) An adaptive knowledge transfer method, called KerKT, that addresses partially overlapping entities - the most common scenario in practice. Extensive experiments were conducted on four real-world datasets, each with three different sparsity ratios. The results show that KerKT alleviates the impact on recommendation caused by data sparsity and transfers knowledge even when there are only a few overlapping entities.

The remainder of the paper is organized as follows. A review of work related to cross-domain recommender systems is provided in Section 2. Section 3 introduces the preliminaries and formally defines the problem to be solved. In Section 4, we present the KerKT method. Section 5 contains the empirical experiments. We evaluated four tasks on four realworld datasets with three data sparsity ratios and three different levels of overlapping entities. The results show that our method performs better in prediction accuracy than six existing nontransfer and cross-domain methods. Lastly, the conclusion and directions for future study are provided in Section 6.

\section{RELATED WORK}

In this section, we review related work on both kernel-based and cross-domain recommender systems.

\section{A. Kernel-based Recommender Systems}

In recommender systems, non-linear interactions between users and items are modeled using kernels. Integrating kernels into a matrix factorization framework, which is a linear combination of the inner product of a user factor matrix and an item factor matrix, provides a more general and more flexible method for updating online models [16]. In [17], Lawrence and Urtasun adopted a Mercer kernel to a non-linear Gaussian Process (GP) model. Ghazanfar et al. [18]incorporated meta data, such as genres and descriptions into the matrix factorization framework, along with kernels 
to solve cold-start problems. Coifman and Lafon [19] used diffusion maps to find representations of data with geometric meanings. Diffusion kernels are a special class of exponential kernels based on the heat equation, which aim to measure the similarities between vertexes or nodes when applied to graphs [20]. However, graph-based diffusion kernel completion is seldom used when dealing with recommendation issues.

\section{B. Cross-domain Recommender Systems}

Cross-domain recommender systems can be divided into three categories: 1) cross-domain recommender systems with side information; 2) cross-domain recommender system with non-overlapping entities; and 3) cross-domain recommender systems with fully-overlapping entities.

1) Cross-domain recommender systems with side information: In this category, it is assumed that some side information about the entities is available. This information might be user-generated information, social information, or the items' attributes. Collective matrix factorization (CMF) [21] is designed for scenarios where a user-item rating matrix and an item-attribute matrix for the same group of items are available. The two matrixes are collectively factorized by sharing the item parameters since the items are the same. The Tagicofi method [22] uses the user-item rating matrix and the user-tag matrix for the same group of users. User similarities extracted from shared tags are used to assist matrix factorization of the original rating matrix. On that basis, TagCDCF [23] extends Tagicofi method to two-domain scenarios, each containing the data of those two matrixes. By integrating the intra-domain and inter-domain correlations and matrix factorization simultaneously, TagCDCF improves recommender system performance in the target domain. In addition to using user-generated tags, hybrid random walk (HRW) [24] bridges cross-domain knowledge through social information.

2) Cross-domain recommender systems with nonoverlapping entities: This category covers methods that handle two domains with non-overlapping entities and transfer knowledge at the group-level. Users and items are clustered into groups and knowledge is shared through grouplevel rating patterns[25]. For example, Codebook Transfer (CBT) [26] clusters users and items into groups and extracts group-level knowledge as a 'codebook'. A probabilistic model, called the rating matrix generated model (RMGM) [27], was subsequently extended from CBT, relaxing the hard membership in groups to soft membership. Neither of these methods ensure that the shared information between two groups in different domains is consistent, so the effectiveness of the knowledge transfer is not guaranteed. Consistent information transfer (CIT) [28] relies on a domain adaptation technique to extract consistent knowledge from the source domain. This method is superior, especially when the data statistics between the source and target domains are divergent.

3) Cross-domain recommender systems with fully overlapping entities: These systems assume that the source and target domains share some common entities. These overlapping entities are used as a bridge, with constraints, to transfer knowledge. Transfer by collective factorization (TCF) [29] was developed to use implicit data in the source domain to help predict explicit feedback in the target domain, such as ratings. However, the assumptions in TCF are very strict - users and items must have a one-to-one mapping across the domains. So, while this method is able to deal with heterogeneous data, its strict assumptions limit the scope for these types of applications in practice. Cross-domain triadic factorization (CDTF) [30] is a user-item-domain tensor that integrates both explicit and implicit feedback. It assumes that users are fully overlapping and that the user factor matrix is the same, thus bridging the domains. Cluster-based matrix factorization (CBMF) [31] tries to extend CDTF to partially overlapping entities, but the core of the CBMF method is the same as for non-overlapping entities, which transfers knowledge based on groups rather than using the overlapping entities as a bridge.

Beyond the cross-domain recommender systems mentioned above, there are some other works related to this study. Since entity correspondence is not always fully available, some strategies have been developed to match users or items across two domains. Li and Lin [32] used latent space matching, to identify unknown user/item mappings. Sometimes, the identifying mappings is time-consuming; hence, Zhao et al. [33] developed an active-learning framework to identify the most valuable correspondences between entities. The process of identifying entity correspondence is not included in this paper, as our problem assumes that only a small number of overlapping entities exists. In summary, methods developed specifically for partially-overlapping entities are rare. In this paper, we introduce KerKT to fill the gap in the literature between fully-overlapping and non-overlapping scenarios.

\section{Preliminaries ANd Problem Formulation}

Matrix factorization is one of the most popular techniques used in recommender systems [34]. In this section, a matrix factorization view of the recommender system in one domain is given to clearly describe the problem setting. The problem under study in this paper is then formulated.

\section{A. A Recommendation Task based on Matrix Factorization in One Domain}

Suppose there are $M$ users and $N$ items in one domain, the relationship between users and items is given as $\boldsymbol{X} \in \mathbb{R}^{M \times N}$ (bold letter represents a matrix). If a user's preferences are represented as ratings, then $\boldsymbol{X}$ is a rating matrix where $\boldsymbol{X}$ is subject to $X_{i j} \in\{1,2,3,4,5, ?\}$ ("?" denotes a missing value). By minimizing its Euclidean distance to the original rating matrix $\boldsymbol{X}$ [34], $\boldsymbol{X}$ is approximated by

$$
\hat{\boldsymbol{X}}=\boldsymbol{U} \boldsymbol{V}^{T}
$$

Thus, $\boldsymbol{U} \in \mathbb{R}^{M \times K}$ is the user feature matrix and $\boldsymbol{V} \in \mathbb{R}^{N \times K}$ is the item feature matrix, which are two low-rank matrixes for users and items, respectively. The $i$ th user and $j$ th item are represented by the $i t h$ and $j t h$ row of the two matrixes as $U_{i *}$ and $V_{j *}$. After matrix factorization, the users and items are mapped to a latent factor feature space of a lower dimensionality $K$. 
The recommendation task is to predict the missing values in the rating matrix based on historical records of the users' preferences. Since the rating matrix $\boldsymbol{X}$ is usually extremely sparse, it is easy to overfit the low-rank approximation matrix factorization. Regularization is usually used on lowrank feature matrixes to avoid this problem. In general, the optimization problem is:

$$
\min _{\boldsymbol{U}, \boldsymbol{V}} \mathcal{L}(f(\boldsymbol{U}, \boldsymbol{V}), \boldsymbol{X})+\lambda \mathcal{R}(\boldsymbol{U}, \boldsymbol{V})
$$

where $\mathcal{L}$ is the loss function of the predicted ratings $f(\boldsymbol{U}, \boldsymbol{V})$ and the original ratings $\boldsymbol{X}, \mathcal{R}(\boldsymbol{U}, \boldsymbol{V})$ is the regularization term, and $\lambda \geqslant 0$ is the regularization trade-off parameter. Similar to probabilistic matrix factorization (PMF), the objective function to measure the loss with regularization terms and a Frobenius norm is [35]:

$$
J(\boldsymbol{U}, \boldsymbol{V})=\frac{1}{2}\left\|\boldsymbol{I} \circ\left(\boldsymbol{X}-\boldsymbol{U} \boldsymbol{V}^{T}\right)\right\|_{F}+\frac{\lambda}{2}\|\boldsymbol{U}\|_{F}+\frac{\lambda}{2}\|\boldsymbol{V}\|_{F}
$$

where $\boldsymbol{I}$ is the rating indicator matrix, $I_{i j} \in\{0,1\} . I_{i j}=1$ indicates that the rating is observed, or $I_{i j}=0$ otherwise. $\circ$ denotes the Hadamard product of the matrixes.

\section{B. Problem Definition}

The problem in this paper is based on the assumption that ratings in the target domain are very sparse. This raises the question of how to use relatively dense data in the source domain to assist a recommendation task in the target domain with overlapping entities. In practice, corresponding entities are not usually easy to identify. Typically, there are many unique entities between different datasets or platforms and only a few common entities. Thus, in this problem setting, the entities partially overlap. Only a small proportion of the entities in the target domain matrix $\boldsymbol{X}_{t}$ have observed correspondences in the source rating matrix $\boldsymbol{X}_{s}$. Even though the entities represent the same user and/or item, the rating a user has given or the rating an item has received can be different in each domain. The overlapping entity indicator matrix is represented by $\boldsymbol{W}^{(s, t)}, W_{i j}^{(s, t)} \in\{0,1\}$. $W_{i j}^{(s, t)}=1$ indicates that the $i$ th entity in the source domain is the same as the $j$ th entity in the target domain, and $W_{i j}^{(s, t)}=0$ otherwise. Without loss of generality, we require the rating rows of overlapping users to be at the top, and the corresponding users are in the same rows in both matrixes. This is achieved by permuting the rows of the original rating matrixes. Thus, the form of the entity indicator matrix $\boldsymbol{W}^{(s, t)}$ is:

$$
\boldsymbol{W}^{(s, t)}=\left[\begin{array}{cc}
\boldsymbol{I}_{o} & 0 \\
0 & 0
\end{array}\right]
$$

where $\boldsymbol{I}_{o}$ is an identity matrix of the same dimension as the number of overlapping entities. This problem of partiallyoverlapping entities in cross-domain recommender systems is formally defined in the following: Given a source rating matrix $\boldsymbol{X}_{s} \in \mathbb{R}^{M_{s} \times N_{s}}$ and a target rating matrix $\boldsymbol{X}_{t} \in \mathbb{R}^{M_{t} \times N_{t}}$, a cross-domain recommender system based on partially overlapping entities is to assist with recommendation task $\hat{\boldsymbol{X}}_{t}=$ $\boldsymbol{U}_{t} \boldsymbol{V}_{t}^{T}$ through an auxiliary source rating matrix $\boldsymbol{X}_{s}$ and an overlapping entity indicator matrix $\boldsymbol{W}^{(s, t)}$.

\section{A CROSS-DOMAIN RECOMMENDER SYSTEM BY KERNEL-INDUCED KNOWLEDGE TRANSFER}

This section introduces our KerKT method. The overlapping entities in each domain may be either users or items. For the purposes of this presentation, we have assumed the users overlap. Overlapping items are handled in the same way and have, therefore, been omitted from this paper. The section begins with an overview of the entire method, then each of the five steps is explained in detail, followed by a small scale example for greater clarity.

\section{A. KerKT Method Overview}

To enable knowledge sharing between the source and target domains with overlapping users, constraints on the user feature matrixes are added to the collective matrix factorization of the source and target rating matrixes. Previous research assumes 'identical' factor matrixes for overlapping entities, but this assumption is too limiting to satisfy in practice. Instead, we have chosen to constrain the similarities between the entities in each domain as a bridge for knowledge transfer. However, while it is easy to measure the similarities between entities in the same domain, inter-domain entity similarities cannot be computed directly.

The overlapping entities are mapped to the same feature space through domain adaptation techniques, while the nonoverlapping entities are connected by diffusion kernel completion. Thus, the similarities between all users in both domains can be measured. Further, constraining the user features using these similarities may lead to a better optimization result. The optimization problem is formalized as:

$$
\min _{\boldsymbol{U}, \boldsymbol{V}} \mathcal{L}(f(\boldsymbol{U}, \boldsymbol{V}), \boldsymbol{X})+\lambda \mathcal{R}(\boldsymbol{U}, \boldsymbol{V})+\lambda_{o} \mathcal{R}_{o}(\boldsymbol{U})
$$

where $\mathcal{R}_{o}(\boldsymbol{U})$ is the regularization term for the entity similarity constraints derived from overlapping users, and $\lambda_{o} \geqslant 0$ is the regularization trade-off parameter.

The KerKT method consists of five steps, as shown in Figure 2. 1). The user features and item features are extracted separately from the source and target domains, and the two sets of user features are aligned to the same feature space through overlapping users. 2). The item features are regulated according to the original rating matrixes and the aligned user feature matrixes. 3). The user and item feature matrixes resulting from the previous two steps are used to measure the user and item similarities in one domain. 4). Kernel-induced completion is conducted to measure the inter-domain user similarities. 5) The user/item features are re-trained based on the constraints of the entity similarities, then recommendations are made. We have selected a specific algorithm to perform each step, but other suitable feature extraction or domain adaptation algorithms could be used as substitutes. The proposed domain adaptation method is contained in Step 1 and 2, while the kernel-induced completion method is contained in Step 3 and 4. The matrix factorization method, with constraints on both intra-domain and inter-domain entity correlations, is contained in Step 5.

\section{B. KerKT Method}

Our proposed KerKT method comprises five steps. 


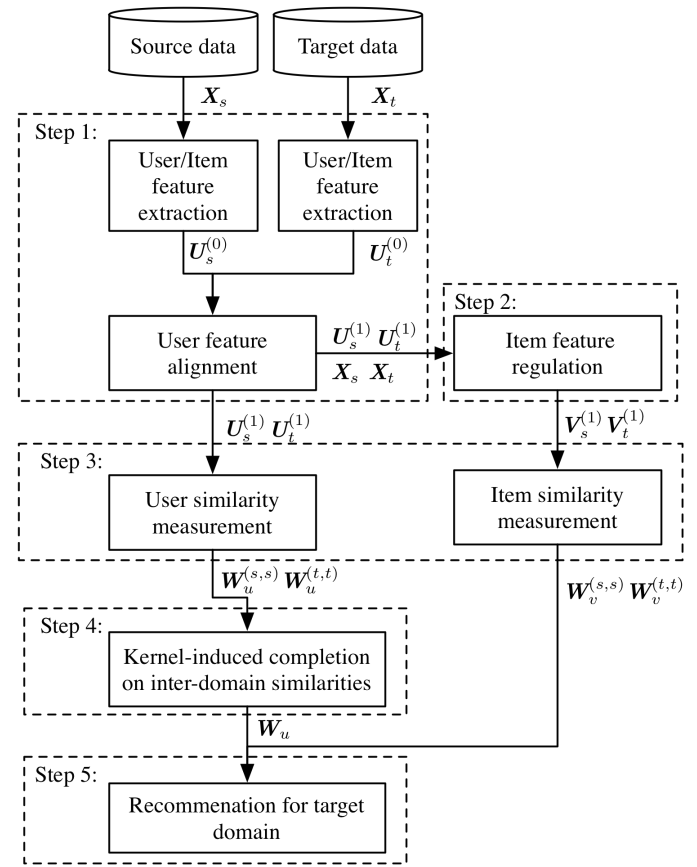

Fig. 2. The procedure of KerKT method.

1) Step 1: Extracting and aligning user features in both domains: In this step, the source rating matrix $\boldsymbol{X}_{s}$ and target rating matrix $\boldsymbol{X}_{t}$ are separately factorized, which results in the user feature matrix $\boldsymbol{U}_{s}$ for the source domain and $\boldsymbol{U}_{t}$ for the target domain. Recall that the users in the source and target domains partially overlap. Accordingly, each user feature matrix can be divided into two parts: one containing the overlapping users; the other containing non-overlapping users. The overlapping user feature matrix for the source domain is denoted as $\boldsymbol{U}_{s, o}$, and $\boldsymbol{U}_{s, n}$ denotes the non-overlapping matrix. The same goes for the target domain i.e. $\boldsymbol{U}_{t, o}$ and $\boldsymbol{U}_{t, n}$.

Assuming the overlapping users have similar tastes or preferences in both domains, we can use them as a bridge to transfer knowledge. However, as mentioned in the Introduction, even the same user's rating patterns may not be completely the same in the two different domains. Data sparsity exacerbates this condition and may lead to two different factorized user feature vectors with different physical meanings. Hence, setting the similarity of the overlapping user entities to 1 may lead to inaccurate similarity measurements, which would eventually negatively impact the effectiveness of the knowledge transfer in the following steps. Therefore, before using the entity correspondences as a strong condition, we need to ensure that the overlapping users in both domains are represented in the same feature space. This is referred to as "subspace alignment" in transfer learning.

The aim is to map the user feature spaces of two overlapping users into a common subspace where domain shift has been eliminated, so the overlapping users ultimately share the same feature space across both domains. In the source domain, the $j$ th column of the overlapping user feature matrix is the representation of the $j$ th user feature. We use a marginal probabilistic distribution of the $j$ th column to represent the characteristics of the user features in each matrix. Thus, the goal is to minimize the differences between the marginal probabilistic distributions of the user features for the source domain and the target domain. If the marginal probability distributions of one user feature are the same in both domains, then the two user features are considered to have the same physical meaning. In this way, we can align the two user feature spaces. In our previous research, we provided a definition for information consistent tri-factorization. However, here, since the scenario and the matrix factorization model are different, this definition has been refined into a definition for consistent matrix factorization with partially overlapping users in the following.

Definition 1 (Consistent Matrix Factorization with Partially overlapping Users). Given a source rating matrix $\boldsymbol{X}_{s} \in$ $\mathbb{R}^{M_{s} \times N_{s}}$ and a target rating matrix $\boldsymbol{X}_{t} \in \mathbb{R}^{M_{t} \times N_{t}}, \boldsymbol{X}_{s}$ and $\boldsymbol{X}_{t}$ can be factorized as follows:

$$
\begin{aligned}
\boldsymbol{X}_{\boldsymbol{s}} & =\left[\begin{array}{l}
\boldsymbol{U}_{s, o} \\
\boldsymbol{U}_{s, n}
\end{array}\right] \boldsymbol{V}_{s}^{T} \\
\boldsymbol{X}_{\boldsymbol{t}} & =\left[\begin{array}{l}
\boldsymbol{U}_{t, o} \\
\boldsymbol{U}_{t, n}
\end{array}\right] \boldsymbol{V}_{t}^{T}
\end{aligned}
$$

where $\boldsymbol{U}_{s, o}$ and $\boldsymbol{U}_{t, o}$ are the overlapping user feature matrixes in the source domain and the target domain, and $\boldsymbol{U}_{s, n}$ and $\boldsymbol{U}_{t, n}$ are the non-overlapping user feature matrixes, respectively.

If both factorizations satisfy the following equation, then they are consistent matrix factorizations:

$$
P\left(\boldsymbol{U}_{s, o}\right)=P\left(\boldsymbol{U}_{t, o}\right)
$$

where $P\left(\boldsymbol{U}_{s, o}\right)$ and $P\left(\boldsymbol{U}_{t, o}\right)$ represent the marginal probability distribution of $\boldsymbol{U}_{s, o}$ and $\boldsymbol{U}_{t, o}$. Thus, the user feature spaces in both the source and target domains are aligned.

To solve a matrix factorization optimization problem that satisfies the above constraints is almost impossible. However, according to Definition 1, a mapping function for those two matrixes can be found to achieve the following equation:

$$
P\left(\boldsymbol{\Psi}_{\boldsymbol{s}}\left(\boldsymbol{U}_{s, o}^{(0)}, \boldsymbol{U}_{t, o}^{(0)}\right)\right)=P\left(\boldsymbol{\Psi}_{\boldsymbol{t}}\left(\boldsymbol{U}_{s, o}^{(0)}, \boldsymbol{U}_{t, o}^{(0)}\right)\right)
$$

A geodesic flow kernel (GFK) is a domain adaptation strategy to find a space that two different feature spaces can be projected into, thus eliminating the divergence of two distributions. We can use this strategy to find a mapping function to align the two user feature spaces formed by overlapping users. Once the GFK operators $\boldsymbol{\Psi}_{\boldsymbol{s}}\left(\boldsymbol{U}_{s}^{(0)}, \boldsymbol{U}_{t}^{(0)}\right)$ are determined, they can be used through the following mapping functions:

$$
\begin{aligned}
& \boldsymbol{\Psi}_{\boldsymbol{s}}\left(\boldsymbol{U}_{s}^{(0)}, \boldsymbol{U}_{t}^{(0)}\right)=\boldsymbol{U}_{s}^{(0)} \times \boldsymbol{\Psi}_{\boldsymbol{G}}\left(\boldsymbol{U}_{s, o}^{(0)}, \boldsymbol{U}_{t, o}^{(0)}\right) \\
& \boldsymbol{\Psi}_{\boldsymbol{t}}\left(\boldsymbol{U}_{s}^{(0)}, \boldsymbol{U}_{t}^{(0)}\right)=\boldsymbol{U}_{t}^{(0)} \times \boldsymbol{\Psi}_{\boldsymbol{G}}\left(\boldsymbol{U}_{s, o}^{(0)}, \boldsymbol{U}_{t, o}^{(0)}\right)
\end{aligned}
$$

where $\boldsymbol{\Psi}_{\boldsymbol{G}}\left(\boldsymbol{U}_{s, o}^{(0)}, \boldsymbol{U}_{t, o}^{(0)}\right)$ are the GFK operators. More details can be found in [28], [36] .

With the divergence eliminated through these mappings, the new representations of the overlapping users will satisfy the conditions in Definition 1. Non-overlapping users also need to be projected onto the same feature space. The mapping functions $\Psi_{s}$ and $\Psi_{t}$ are used for this purpose.

$$
\begin{aligned}
& \boldsymbol{U}_{s}^{(1)}=\boldsymbol{\Psi}_{\boldsymbol{s}}\left(\boldsymbol{U}_{s}^{(0)}, \boldsymbol{U}_{t}^{(0)}\right) \\
& \boldsymbol{U}_{t}^{(1)}=\boldsymbol{\Psi}_{\boldsymbol{t}}\left(\boldsymbol{U}_{s}^{(0)}, \boldsymbol{U}_{t}^{(0)}\right)
\end{aligned}
$$


where $\boldsymbol{U}_{s}^{(1)}$ and $\boldsymbol{U}_{t}^{(1)}$ are the aligned user feature matrixes after mapping, and $\boldsymbol{\Psi}_{s}$ and $\boldsymbol{\Psi}_{\boldsymbol{t}}$ are the mapping functions using GFK.

How the new user feature spaces are derived and how $\boldsymbol{U}_{s}^{(1)}$ and $\boldsymbol{U}_{t}^{(1)}$ are learned is summarized in Algorithm 1.

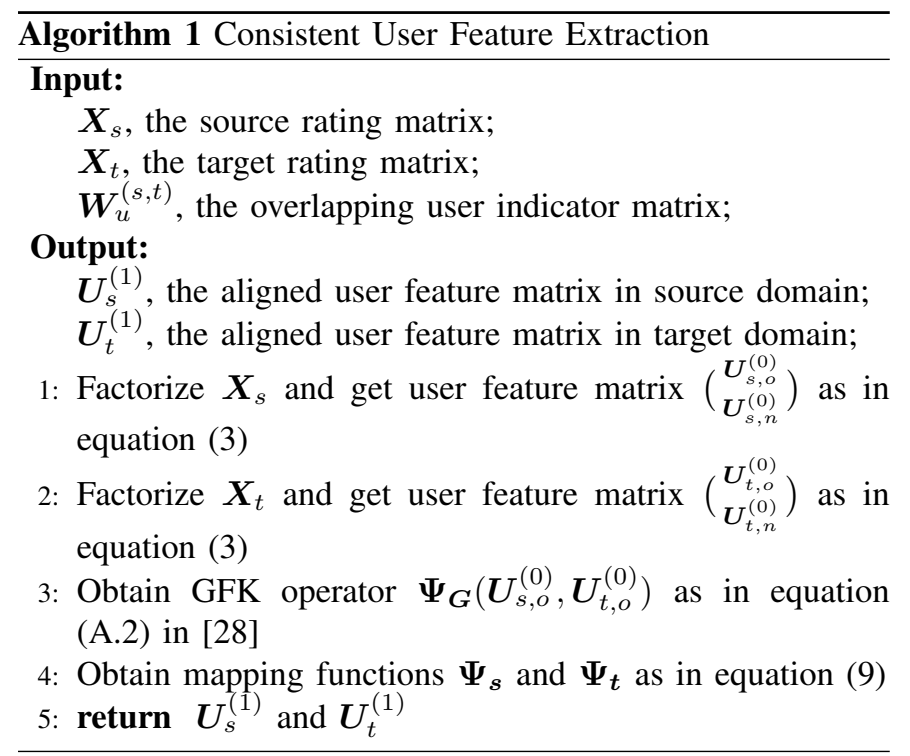

2) Step 2: Item feature regulation in both domains: In matrix factorization, the user feature matrix and the item feature matrix are both low-rank matrixes that map users and items to the same $k$-dimensional feature space. So, once the user feature spaces are aligned, the item feature matrixes should be regularized to the new $k$-dimensional feature space. The new item feature matrixes are obtained by minimizing the distance between the approximations of the low-rank matrixes and the original data in the rating matrix. A Frobenius norm is used to measure the distance. The cost function of source domain matrix follows, the target domain matrix has the same form:

$J_{v}\left(\boldsymbol{V}_{s}^{(1)}\right)=\frac{1}{2}\left\|\boldsymbol{I}_{\boldsymbol{s}} \circ\left(\boldsymbol{X}_{s}-\boldsymbol{U}_{s}^{(1)}\left(\boldsymbol{V}_{s}^{(1)}\right)^{T}\right)\right\|_{F}+\frac{\lambda_{V_{s}}}{2}\left\|\boldsymbol{V}_{s}^{(1)}\right\|_{F}$

where $\lambda_{V_{s}}$ is the regularization parameter. The item feature matrixes are learned by optimizing:

$$
\min J_{v}\left(\boldsymbol{V}_{s}^{(1)}\right)
$$

Gradient descent is used for this optimization. The update rule is:

$$
\boldsymbol{V}_{s}^{(1)} \leftarrow \boldsymbol{V}_{s}^{(1)}-\eta_{V_{s}}\left[\left(\boldsymbol{U}_{s}^{(1)}\left(\boldsymbol{V}_{s}^{(1)}\right)^{T}-\boldsymbol{X}_{s}\right) \boldsymbol{U}_{s}^{(1)}+\lambda_{V_{s}} \boldsymbol{V}_{s}^{(1)}\right]
$$

where the learning rate is $\eta_{V_{s}} . V_{t}^{(1)}$ can be obtained through the same process.

This step is summarized in Algorithm 2.

3) Step 3: Entity similarity measures in one domain: This step calculates the user and item similarities in one domain. Since the rating matrix is very sparse, making this calculation directly from the rating matrix can lead to inaccurate results. Hence, using the PMF formulation introduced in Section III-A, one rating $X_{i j}$ is generated from a user latent feature vector $U_{i *}$ and an item latent feature vector

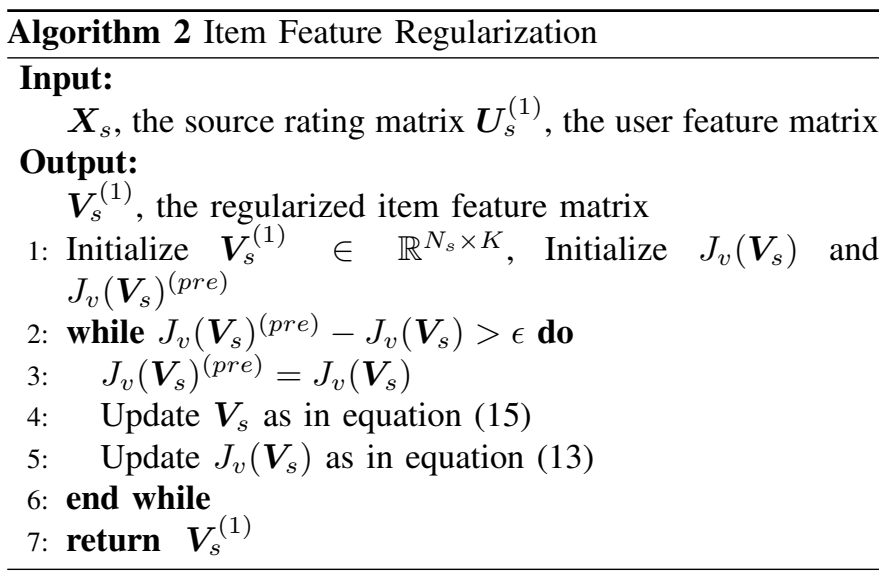

$V_{* j}$. Thus, the source rating matrix and target rating matrix can be factorized as $\boldsymbol{X}_{s}=\boldsymbol{U}_{s} \boldsymbol{V}_{s}^{T}$ and $\boldsymbol{X}_{t}=\boldsymbol{U}_{t} \boldsymbol{V}_{t}^{T}$. This is a dimensionality reduction and data compression process as users/items are mapped to a lower $k$-dimensional feature space (usually $k \ll M, k \ll N$ ). Once complete, users and items are represented as full $k$-dimensional feature matrixes, and the user/item similarities can be calculated from the user/item feature matrixes.

Similarity measurements are easy with user and item feature spaces in one domain since the feature spaces are homogeneous. And there are many suitable choices for performing these calculations, such as cosine similarity, Pearson's similarity, Euclidean measurement, or the RBF measurement. The choice depends on the situation and the characteristics of the domain. For example, cosine similarity is very popular and effective for word count and text similarity measurements due to the advantages of using angles rather than distance. Pearson's measurement tends to be more effective in memorybased collaborative filtering methods owing to its emphasis on averages. In this problem, we are measuring user similarity from a user feature matrix where the feature values are real numbers, so the following RBF measurement is the most appropriate: $W_{i j}=e^{-\frac{\left\|U_{i *}-U_{j *}\right\|^{2}}{\sigma^{2}}}$, where $\sigma^{2}$ is set to be the median of all the non-zero values calculated by $\left\|U_{i *}-U_{j *}\right\|^{2}$.

4) Step 4: Kernel induced completion of inter-domain user similarity: In inter-domain user similarity measurement, the user feature spaces are not the same and the user features are heterogeneous, which means their similarities cannot be calculated directly. However, given the first three steps, some user similarities between the source and target domains are now known. The overlapping entity indicator matrix $\boldsymbol{W}^{(s, t)}$ contains the observed overlapping user information. Hence, a full user similarity matrix can be constructed as:

$$
\boldsymbol{W}_{u}=\left[\begin{array}{ll}
\boldsymbol{W}_{u}^{(s, s)}, & \boldsymbol{W}_{u}^{(s, t)} \\
\boldsymbol{W}_{u}^{(t, s)}, & \boldsymbol{W}_{u}^{(t, t)}
\end{array}\right]
$$

where $\boldsymbol{W}_{u}^{(s, s)}$ and $\boldsymbol{W}_{u}^{(t, t)}$ represent the user similarities in the source and target domains, respectively, and $\boldsymbol{W}_{u}^{(s, t)}=$ $\left(\boldsymbol{W}_{u}^{(t, s)}\right)^{T}$ represents the inter-domain user similarities.

As in Step 2, the two feature spaces of the overlapping users are aligned to eliminate feature space divergence. Therefore, it is reasonable to set the similarity of observed overlapping 
users to $\left(\boldsymbol{W}_{u}^{(s, t)}\right)_{i j}=1$ in $\boldsymbol{W}^{(s, t)}$. For now, the similarities between the overlapping users are the only known entries in the inter-domain similarity matrix. We need to complete $\boldsymbol{W}_{u}^{(s, t)}$ using the information from $\boldsymbol{W}_{u}$. Note that, here, the non-overlapping users and their features are heterogeneous. Thus, their similarities cannot be computed directly.

This matrix completion problem has a strong connection to a bipartite edge completion problem [37]. In Step 3, the user similarities are all measured within one domain. As a result, we have fully connected nodes in the graph representations of both the source and target domains, as indicated by the red and blue nodes in Figure 3. The overlapping users are shown as purple nodes in the graph, and they act as "bridge" to couple the two graphs. To complete the user similarity matrix $\boldsymbol{W}_{u}$ requires filling in all the edges from the entire graph. The subscript $u$ has been omitted to simplify the notation.

In network propagation, a random walk is a good way to reach to all the nodes. As shown in Figure 3, one user entity, denoted as a node $x$ in the source domain, is fully connected with all the other node in the source domain $W_{x p}^{(s, s)}, p \in \mathcal{U}_{s}$, so does node $y$ in the target domain $W_{y q}^{(t, t)}, q \in \mathcal{U}_{t}$. If node $p$ in the source domain and node $q$ in the target domain are overlapping users, i.e. they are the same user, then $W_{p q}^{(s, t)}=1$. The two nodes $x$ and $y$ are connected and their similarity can be calculated as: $W_{x y}^{(s, t)} \leftarrow W_{x p}^{(s, s)} W_{p q}^{(s, t)} W_{y q}^{(t, t)}$. By aggregating all the nodes connected to $x$ in the source domain and $y$ in the target domain, the edge can be completed with: $W_{x y}^{(s, t)} \leftarrow \sum_{p \in U_{s}} \sum_{q \in U_{t}} W_{x p}^{(s, s)} W_{p q}^{(s, t)} W_{y q}^{(t, t)}$. In a matrix form, this is written as:

$$
\boldsymbol{W}^{(s, t),(1)}=\boldsymbol{W}^{(s, s)} \boldsymbol{W}^{(s, t)} \boldsymbol{W}^{(t, t)}
$$

The above equation can be treated as a one-step random walk from both the source domain and the target domain. Generally, $M$ steps of random walk are taken in total from the source and target sides, and all the possible steps are added together to complete the final graph:

$$
\boldsymbol{W}^{(s, t),(K)}=\sum_{K=0}^{M}\left(\begin{array}{l}
M \\
K
\end{array}\right)\left(\boldsymbol{W}^{(s, s)}\right)^{K} \boldsymbol{W}^{(s, t)}\left(\boldsymbol{W}^{(t, t)}\right)^{M-K}
$$

However, the goal in this problem is to find all the similarities between all the users in both domains. Therefore, a finite number of random walk steps may not identify all the possible relationships, but it would be more likely to associate all the indirectly connected users if $K$ was infinite. Hence, the diffusion kernel completion method [38] is used to complete the user similarity matrix:

$$
\boldsymbol{W}^{(s, t)}=e^{\left(\beta_{s} \boldsymbol{W}^{(s, s)}\right)} \boldsymbol{W}^{(s, t)} e^{\left(\beta_{t} \boldsymbol{W}^{(t, t)}\right)}
$$

where $\beta_{s}$ and $\beta_{t}$ are two positive scalars to regulate the weights of the source and the target domains.

5) Step 5: Collective matrix factorization with user similarity constraints: With all similarities measured and all the pairs of nodes connected, a fully connected graph can be constructed. A very common strategy for increasing computational speed is to remove edges, leave a sparse graph. This

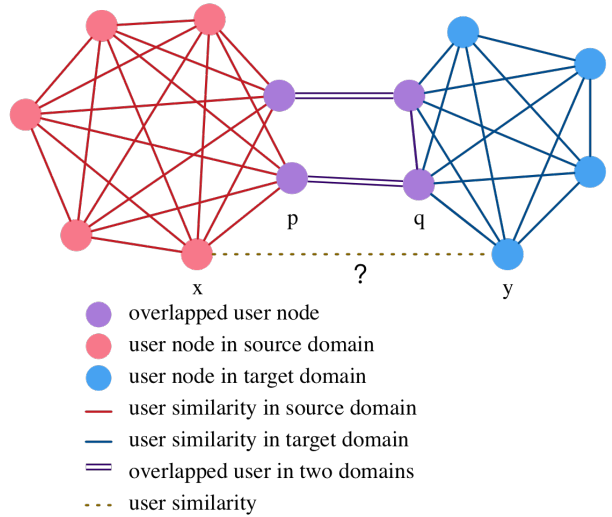

Fig. 3. Graphical view of user relationships in source and target domains

approach tends to achieve better performance empirically as it emphasizes local information with high similarity while ignoring information that is likely to be false. Hence, the $k$ nearest neighbors of each node are retained in a similar way to the original memory-based collaborative filtering strategy.

In the scenario of this paper, only overlapping users are observed but items are non-overlapping. The users have both intra-domain and inter-domain similarities, but the items only have intra-domain similarities. Based on the assumption that "similar users have similar tastes and will thus choose similar items to consume", both the intra-domain and inter-domain similarities are used to constrain the proposed matrix factorization as prior knowledge. In terms of intra-domain similarities, although the data in the target domain are very sparse, they are still very valuable for measuring the similarities between users/items so as to constrain the matrix factorization. As for inter-domain similarities, users in target domain are not only correlated to users in their own domain but also in the source domain via the overlapping users. As a consequence, users in the source domain with similar preferences to users in the target domain are transferred as knowledge to improve the performance of the recommender system.

The constraints result in users who are similar tend to have similar latent factors. We have used the regularization form in [22]:

$$
\mathcal{R}_{o}(\boldsymbol{U})=\operatorname{tr}\left(\boldsymbol{U}^{T} \boldsymbol{L} \boldsymbol{U}\right)
$$

where $\boldsymbol{L}$ denotes a Laplacian matrix, and $\boldsymbol{L}=\boldsymbol{D}-\boldsymbol{W} . \boldsymbol{W}$ is the user similarity matrix, and $\boldsymbol{D}$ is a diagonal matrix defined as $D_{i i}=\sum_{j} W_{i j}$.

Note that, although the form of regularization is quite similar, our method is different to [33]. In [33], the similarities between entities in the source domain are directly used in the target domain as prior knowledge without considering the inconsistencies between the features in each domain. By contrast, in our method, the similarities between entities in target domain are learned through the domain adaption technique, which ensures the features are consistent, and through the diffusion kernel technique, which makes the similarity constraints more accurate and complete. Our proposed constraints are both more flexible and more reasonable to satisfy in practice. 
This goal is achieved by minimizing the following objective function:

$$
\begin{aligned}
f\left(\boldsymbol{U}_{\boldsymbol{s}}, \boldsymbol{V}_{\boldsymbol{s}}, \boldsymbol{U}_{\boldsymbol{t}}, \boldsymbol{V}_{\boldsymbol{t}}\right) & =\frac{\alpha}{2}\left\|\boldsymbol{I}_{s} \circ\left(\boldsymbol{X}_{s}-\boldsymbol{U}_{s} \boldsymbol{V}_{s}^{T}\right)\right\|_{F} \\
& +\frac{1}{2}\left\|\boldsymbol{I}_{t} \circ\left(\boldsymbol{X}_{t}-\boldsymbol{U}_{t} \boldsymbol{V}_{t}^{T}\right)\right\|_{F} \\
& +\frac{\lambda_{u}}{2}\left[\operatorname{tr}\left(\boldsymbol{U}_{s}^{T} \boldsymbol{L}_{u}^{(s, s)} \boldsymbol{U}_{s}\right)+\operatorname{tr}\left(\boldsymbol{U}_{s}^{T} \boldsymbol{L}_{u}^{(s, t)} \boldsymbol{U}_{t}\right)\right. \\
& \left.+\operatorname{tr}\left(\boldsymbol{U}_{t}^{T}\left(\boldsymbol{L}_{u}^{(s, t)}\right)^{T} \boldsymbol{U}_{s}\right)+\operatorname{tr}\left(\boldsymbol{U}_{t}^{T} \boldsymbol{L}_{u}^{(t, t)} \boldsymbol{U}_{t}\right)\right] \\
& +\frac{\lambda_{v}}{2}\left[\operatorname{tr}\left(\boldsymbol{V}_{s}^{T} \boldsymbol{L}_{v}^{(s, s)} \boldsymbol{V}_{s}\right)+\operatorname{tr}\left(\boldsymbol{V}_{t}^{T} \boldsymbol{L}_{v}^{(t, t)} \boldsymbol{V}_{t}\right)\right] \\
& +\frac{\lambda}{2}\left(\left\|\boldsymbol{U}_{s}\right\|_{F}+\mid \boldsymbol{V}_{s}\left\|_{F}+\right\| \boldsymbol{U}_{t}\left\|_{F}+\right\| \boldsymbol{V}_{t} \|_{F}\right)
\end{aligned}
$$

where $t r$ is the trace of a matrix, $\alpha \in(0,1)$ is trade-off parameter to balance the source and target domain data, and $\lambda_{u}, \lambda_{u}$ and $\lambda_{u}$ are the regularization parameters to control the influence of the constraints on the user similarities, item similarities and algorithm complexity. Details on how these parameters affect the proposed method are presented in Section $\mathrm{V}$. Using gradient descent, the objective function is minimized with the following update rules:

$$
\begin{aligned}
& \boldsymbol{U}_{s} \leftarrow \boldsymbol{U}_{s}-\eta_{U_{s}}\left[\alpha\left(\boldsymbol{U}_{s} \boldsymbol{V}_{s}^{T}-\boldsymbol{X}_{s}\right) \boldsymbol{V}_{s}+\right. \\
& \left.\quad \lambda_{u} \boldsymbol{L}_{u}^{(s, s)} \boldsymbol{U}_{s}+\frac{\lambda_{u}}{2} \boldsymbol{L}_{u}^{(s, t)} \boldsymbol{U}_{t}+\lambda \boldsymbol{U}_{s}\right] \\
& \boldsymbol{V}_{s} \leftarrow \boldsymbol{V}_{s}-\eta_{V_{s}}\left[\alpha\left(\boldsymbol{V}_{s} \boldsymbol{U}_{s}^{T}-\boldsymbol{X}_{s}^{T}\right) \boldsymbol{U}_{s}+\lambda_{v} \boldsymbol{L}_{v}^{(s, s)} \boldsymbol{V}_{s}+\lambda \boldsymbol{U}_{s}\right] \\
& \boldsymbol{U}_{t} \leftarrow \boldsymbol{U}_{t}-\eta_{U_{t}}\left[\left(\boldsymbol{U}_{t} \boldsymbol{V}_{t}^{T}-\boldsymbol{X}_{t}\right) \boldsymbol{V}_{t}+\right. \\
& \left.\quad \lambda_{u} \boldsymbol{L}_{u}^{(t, t)} \boldsymbol{U}_{t}+\frac{\lambda_{u}}{2}\left(\boldsymbol{L}_{u}^{(s, t)}\right)^{T} \boldsymbol{U}_{s}+\lambda \boldsymbol{U}_{t}\right] \\
& \boldsymbol{V}_{t} \leftarrow \boldsymbol{V}_{t}-\eta_{V_{t}}\left[\left(\boldsymbol{V}_{t} \boldsymbol{U}_{t}^{T}-\boldsymbol{X}_{t}^{T}\right) \boldsymbol{U}_{t}+\lambda_{v} \boldsymbol{L}_{v}^{(t, t)} \boldsymbol{V}_{t}+\lambda \boldsymbol{U}_{t}\right]
\end{aligned}
$$

By updating $\boldsymbol{U}_{s}, \boldsymbol{V}_{s}, \boldsymbol{U}_{t}$ and $\boldsymbol{V}_{t}$ iteratively, we arrive at a final optimized approximation of $\hat{\boldsymbol{X}}_{t}=\boldsymbol{U}_{t} \boldsymbol{V}_{t}^{T}$. Recommendations are given according to the rating prediction in the target domain.

\section{A Small-scale Example}

To better illustrate our method, this subsection outlines a small-scale example. Suppose the source domain of a recommender system contains five users, denoted as $\mathcal{U}_{s}=$ $\left\{u_{1}, u_{2}, u_{3}, u_{4}, u_{5}\right\}$, and nine items, denoted as $\mathcal{I}_{s}=$ $\left\{i_{1}, i_{2}, i_{3}, i_{4}, i_{5}, i_{6}, i_{7}, i_{8}, i_{9}\right\}$. The target domain contains six users, denoted as $\mathcal{U}_{t}=\left\{u_{6}, u_{7}, u_{8}, u_{9}, u_{10}, u_{11}\right\}$, and nine items, denoted as $\mathcal{I}_{t}=\left\{j_{1}, j_{2}, j_{3}, j_{4}, j_{5}, j_{6}, j_{7}, j_{8}, j_{9}\right\}$. Four of the nine items in the source domain $i_{6}, i_{7}, i_{8}, i_{9}$ correspond to four of the nine items in the target domain - $j_{1}, j_{2}, j_{3}, j_{4}$ respectively as shown in Fig. 4.

Step 1: Extracting and aligning features: With the lowerdimension $K$ set to 4 , the source rating matrix $\boldsymbol{X}_{s}$ and target rating matrix $\boldsymbol{X}_{t}$ are factorized into user feature matrix and item feature matrix according to (5) respectively. Then, the features of the overlapping items are projected onto the same feature space using GFK. $\boldsymbol{V}_{s, o}$ and $\boldsymbol{V}_{t, o}$ are used to find the proper projection matrix, and the item feature matrixes $\boldsymbol{V}_{s}^{(0)}$
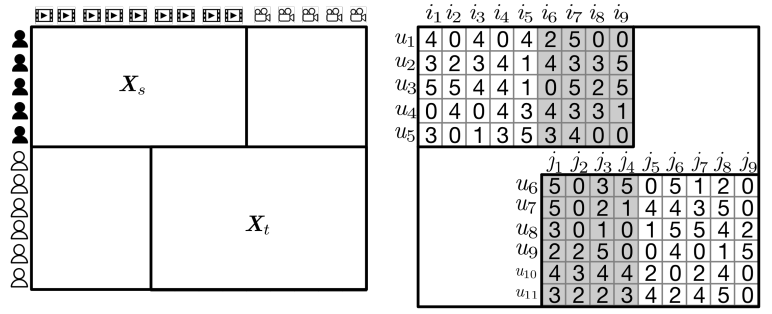

Fig. 4. A small scale example.
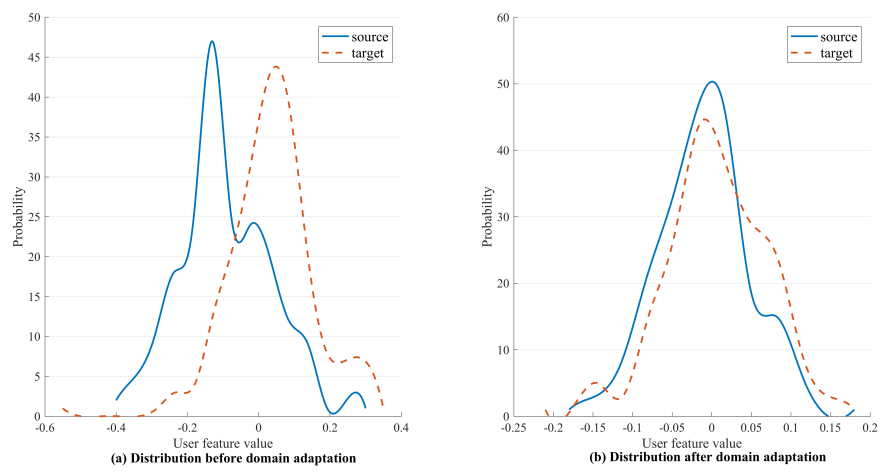

Fig. 5. An example of domain adaptation on user feature matrix with Netflix dataset.

and $\boldsymbol{V}_{t}^{(0)}$ are transformed into $\boldsymbol{V}_{s}^{(1)}$ and $\boldsymbol{V}_{t}^{(1)}$. The example in Fig. 5 illustrates the domain adaptation process using GFK. In this small-scale example, there is not sufficient data to generate meaningful distribution. Therefore, we have used the result and item feature matrixes from our Task 2 experiment in Section V. Fig. 5 (a) shows the distribution of one item feature for the items that overlap in the source and target domains. It is apparent that a divergence exists between these two distributions. Fig. 5 (b) shows the adjustment to the feature after domain adaptation. Here, the feature distributions for the source domain and the target domain are far better aligned.

Step 2: Item feature regulation: $\boldsymbol{U}_{s}^{(1)}$ and $\boldsymbol{U}_{t}^{(1)}$ are updated according to (13).

Step 3: Similarity measures: This step shows how the item similarity matrix $\boldsymbol{W}_{v}$ is calculated, given that item overlaps exist in our example. With item feature matrixes from the source domain and the target domain, the item similarity in the same domain can be calculated directly through RBF measurement. Together with the overlap information, the item similarity matrix $\boldsymbol{W}_{v}$ is

$$
\boldsymbol{W}_{v}=\left[\begin{array}{ll}
\boldsymbol{W}_{v}^{(s, s)}, & \boldsymbol{W}_{v}^{(s, t)} \\
\boldsymbol{W}_{v}^{(t, s)}, & \boldsymbol{W}_{v}^{(t, t)}
\end{array}\right]
$$

where

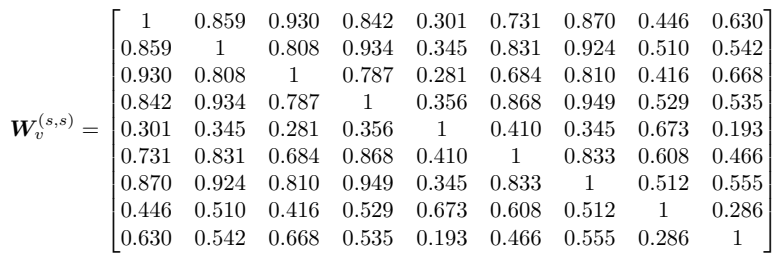




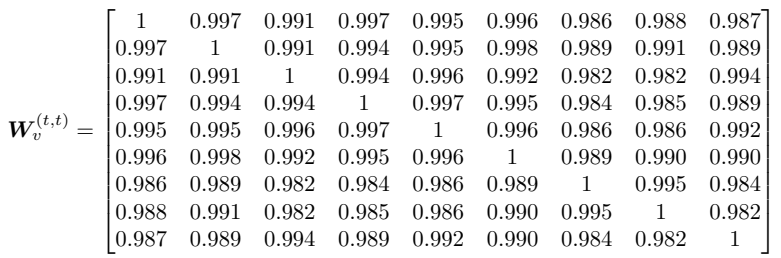

$\boldsymbol{W}_{v}^{(s, t)}=\left(\boldsymbol{W}_{v}^{(t, s)}\right)^{T}=\left[\begin{array}{ccccccccc}0 & 0 & 0 & 0 & 0 & 0 & 0 & 0 & 0 \\ 0 & 0 & 0 & 0 & 0 & 0 & 0 & 0 & 0 \\ 0 & 0 & 0 & 0 & 0 & 0 & 0 & 0 & 0 \\ 0 & 0 & 0 & 0 & 0 & 0 & 0 & 0 & 0 \\ 0 & 0 & 0 & 0 & 0 & 0 & 0 & 0 & 0 \\ 1 & 0 & 0 & 0 & 0 & 0 & 0 & 0 & 0 \\ 0 & 1 & 0 & 0 & 0 & 0 & 0 & 0 & 0 \\ 0 & 0 & 1 & 0 & 0 & 0 & 0 & 0 & 0 \\ 0 & 0 & 0 & 1 & 0 & 0 & 0 & 0 & 0\end{array}\right]$

Step 4: Kernel induced completion: The similarity matrix $\boldsymbol{W}_{v}^{(s, t)}$ is completed according to (19). In more detail, the low-rank eigen-decomposition for both $\boldsymbol{W}_{v}^{(s, s)}$ and $\boldsymbol{W}_{v}^{(t, t)}$ is:

$$
\begin{aligned}
e^{\left(\beta_{s} \boldsymbol{W}_{v}^{(s, s)}\right)} & \approx \boldsymbol{Q}_{s} e^{\left(\beta_{s} \boldsymbol{D}_{s}\right)} \boldsymbol{Q}_{s}^{T} \\
e^{\left(\beta_{t} \boldsymbol{W}_{v}^{(t, t)}\right)} & \approx \boldsymbol{Q}_{t} e^{\left(\beta_{t} \boldsymbol{D}_{t}\right)} \boldsymbol{Q}_{t}^{T}
\end{aligned}
$$

where $\boldsymbol{D}_{s}$ and $\boldsymbol{D}_{t}$ are the $k_{s}, k_{t}$ leading eigen-values for $\boldsymbol{W}_{v}^{(s, s)}$ and $\boldsymbol{W}_{v}^{(t, t)}$ and $\boldsymbol{Q}_{s}$ and $\boldsymbol{Q}_{t}$ are the corresponding eigenvalues. Diffusion completion is then conducted as

$$
\hat{\boldsymbol{W}}_{v}^{(s, t)}=\left(\boldsymbol{Q}_{s} e^{\left(\beta_{s} \boldsymbol{D}_{s}\right)} \boldsymbol{Q}_{s}^{T}\right) \boldsymbol{W}_{v}^{(s, t)}\left(\boldsymbol{Q}_{t} e^{\left(\beta_{t} \boldsymbol{D}_{t}\right)} \boldsymbol{Q}_{t}^{T}\right)
$$

The final result of $\hat{\boldsymbol{W}}_{v}^{(s, t)}$ in this example is:

$\hat{\boldsymbol{W}}_{v}^{(s, t)}=\left[\begin{array}{ccccccccc}0 & 0 & 0 & 0.003 & 0 & 0 & 0 & 0 & 0 \\ 0.004 & 0.004 & 0 & 0 & 0 & 0 & 0 & 0 & 0 \\ 0 & 0 & 0 & 0.003 & 0 & 0 & 0 & 0 & 0 \\ 0.004 & 0.005 & 0.002 & 0 & 6.7 \times 10^{-5} & 6.7 \times 10^{5} & 6.7 \times 10^{5} & 6.7 \times 10^{5} & 6.7 \times 10^{5} \\ 0 & 0 & 0.003 & 0 & 0 & 0 & 0 & 0 & 0 \\ 1 & 0.009 & 0.008 & 0.007 & 0.005 & 0.005 & 0.005 & 0.005 & 0.005 \\ 0.009 & 1 & 0.007 & 0.007 & 0.005 & 0.005 & 0.005 & 0.005 & 0.005 \\ 0.008 & 0.007 & 1 & 0.006 & 0.005 & 0.005 & 0.005 & 0.005 & 0.005 \\ 0.007 & 0.007 & 0.006 & 1 & 0.005 & 0.005 & 0.005 & 0.005 & 0.005\end{array}\right]$

Step 5: Collective matrix factorization: With the user and item similarity matrix available, the target rating matrix is approximated as indicated in (21) and recommendations can be given accordingly.

\section{EXPERIMENTS AND ANALYSIS}

This section presents the experimental results and related analysis. The datasets and evaluation metrics are introduced first, followed by the experimental settings and baseline methods. Then, we present the results of the empirical experiments, with a parameter analysis to conclude the section.

\section{A. Datasets and Evaluation Metrics}

Our method was tested under the conditions that the source and target domains share some overlapping users and/or items. For a fair comparison, we chose movies and books as the recommendation subject - two commonly-used categories in previous research on cross-domain recommender systems. Four real-world datasets were used in our experiments: Movielens ${ }^{1}$, Netflix ${ }^{2}$, AmazonBook ${ }^{3}$ [39] and Douban ${ }^{4}$ [40]. Each of these datasets is publicly available and has been tested on

\footnotetext{
${ }^{1}$ https://grouplens.org/datasets/movielens/20m/

${ }^{2}$ https://netflixprize.com/index.html

${ }^{3}$ http://jmcauley.ucsd.edu/data/amazon

${ }^{4}$ https://sites.google.com/site/erhengzhong/datasets
}

TABLE I

STATISTICS OF ORIGINAL DATASETS

\begin{tabular}{llllll}
\hline & Movielens20M & Netflix & Amazon_book & Douban_movie & Douban_book \\
\hline \#user & 138493 & 480189 & 8026324 & 28718 & 26877 \\
\#item & 26744 & 17770 & 2330066 & 57424 & 187520 \\
\#rating & 20000263 & 100480507 & 22507155 & 2828585 & 1097148 \\
sparsity & $0.54 \%$ & $1.18 \%$ & $0.0001 \%$ & $0.17 \%$ & $0.02 \%$ \\
rating_range & $0.5-5$ & $1-5$ & $1-5$ & $1-5$ & $1-5$ \\
\hline
\end{tabular}

single domain recommendation in a variety of situations, but rarely in cross-domain recommendation. Our experiments are a supplement to the lack of tests in this specific problem setting. The statistical information for these datasets is presented in Table I.

From AmazonBooks, we removed all users who had given exactly the same rating for every book, as these data are not effective for constructing a recommender system [28]. Movielens20M was normalized to the range of $\{1,2,3,4,5\}$. Four cross-domain recommendation tasks were designed for experiments: Task 1: movie $\rightarrow$ movie, user-overlap, Movielens20M; Task 2: movie $\rightarrow$ movie, item-overlap, Netflix; Task 3: book $\rightarrow$ book, item-overlap, AmazonBook; Task 4: movie $\rightarrow$ book, user-overlap, Douban.

In the first three tasks, we used the data from one dataset and split the entities into the source domain and the target domain to simulate entity overlaps. The fourth task was designed for Douban, a real-world rating website where users can rate movies, books, and music. We now turn to Task 1 as an example to describe how the data was selected. The process for Task 2 and Task 3 was similar but with overlapping items. With the source domain data, we filtered out the users who had given less than a total of 20 ratings and items who had received less than 10 ratings. We randomly selected 2000 items and 2000 users, constraining the sparsity to $2 \%$ to ensure the source domain data were relatively dense. In the 2000 users, we randomly chose 200 users as overlapping users. We then randomly selected 1800 users with no correspondence to the 2000 users in the source domain. In total, these users composed the 2000 users in the target domain data. Additionally, we also randomly chose 2000 items for the target domain data that had no intersections with items in the source domain. We tested the target domain data with three sparsity ratios to compare the different algorithms in different circumstances. The details of the final datasets are summarized in Table II.

Mean Absolute Error (MAE) and Root Mean Square Error (RMSE) were used as evaluation metrics:

$$
\begin{aligned}
& M A E=\sum_{u, v, X_{u v} \in Y} \frac{\left|\hat{X}_{u v}-X_{u v}\right|}{|Y|} \\
& R M S E=\sqrt{\sum_{u, v, X_{u v} \in Y} \frac{\left(\hat{X}_{u v}-X_{u v}\right)^{2}}{|Y|}}
\end{aligned}
$$

where $X_{u v}$ and $\hat{X}_{u v}$ are the true and predicted ratings, $Y$ is the test set and $|Y|$ is the number of the test set. The smaller the errors, the better the performance. 
TABLE II

DESCRIPTION OF DATA SUBSETS FOR FOUR TASKS.

\begin{tabular}{lllllc}
\hline Task & Data_name & Data_source & Domain & Sparsity & No. of overlapping \\
\hline \multirow{4}{*}{ Task 1 } & task1_s1 & Movielens20M & source & $2.00 \%$ & $200,100,50$ \\
& task1_t1 & Movielens20M & target & $0.50 \%$ & $200,100,50$ \\
& task1_t2 & Movielens20M & target & $1.00 \%$ & $200,100,50$ \\
& task1_t3 & Movielens20M & target & $1.50 \%$ & $200,100,50$ \\
& task2_s1 & Netflix & source & $2.00 \%$ & $200,100,50$ \\
Task 2 & task2_t1 & Netflix & target & $0.50 \%$ & $200,100,50$ \\
& task2_t2 & Netflix & target & $1.00 \%$ & $200,100,50$ \\
& task2_t3 & Netflix & target & $1.50 \%$ & $200,100,50$ \\
& & & & & \\
& task3_s1 & AmazonBook & source & $2.00 \%$ & $200,100,50$ \\
Task 3 3 & task3_t1 & AmazonBook & target & $0.50 \%$ & $200,100,50$ \\
& task3_t2 & AmazonBook & target & $0.63 \%$ & $200,100,50$ \\
& task3_t3 & AmazonBook & target & $0.75 \%$ & $200,100,50$ \\
& task4_s1 & DoubanMovie & source & $2.00 \%$ & $200,100,50$ \\
Task 4 4 & task4_t1 & DoubanBook & target & $0.50 \%$ & $200,100,50$ \\
& task4_t2 & DoubanBook & target & $1.00 \%$ & $200,100,50$ \\
& task4_t3 & DoubanBook & target & $1.50 \%$ & $200,100,50$ \\
\hline & & & & & \\
\hline
\end{tabular}

\section{B. Experimental Settings and Baselines}

Three non-transfer learning methods were chosen for comparison: Pearson's correlation coefficient (PCC) [41], flexible mixture model (FMM) [42] and PMF [35], along with three cross-domain recommendation methods, CBT [26], RMGM [27], and probabilistic matrix factorization transfer learning (PMFTL) [33]. PCC is a classical memory-based collaborative filtering method. FMM is a graphical model designed to allow one user/item to be clustered into several groups simultaneously. Empirically, FMM has been proven to be more effective in providing recommendations to users with few historical ratings. RMGM is a cross-domain recommendation method that evolved out of the single domain FMM. CBT is also a cross-domain recommendation method. Both of these methods were designed for scenarios with no overlapping users or items. PMFTL is a transfer learning method for cross-domain scenarios with entity overlap as proposed in [33]; However, for a fair comparison, we removed the active learning module in the originally proposed method. PMFTL was developed on the basis of PMF with partially overlapping entities. PMFTL has more relaxed constraints than TCF [29]. TCF was designed for problems where users and items have a one-to-one mapping. The constraints in TCF are strict. One constraint requires that the user and item feature matrixes in the source and target domains are exactly the same. Whereas, PMFTL uses similarities estimated in the source domain directly as constraints in the target domain. Since TCF cannot be used to solve the problem presented in this paper, we did not select it for comparison.

User-based collaborative-filtering was used for PCC, with the number of neighboring users set to 50. For FMM, CBT and RMGM, the number of user and item groups were both set to 50. For PMF and PMFTL, we set $\lambda=$ $\{0.01,0.05,0.1,0.2,0.3,0.5,1\}$. The parameter settings for KerKT were $\alpha=0.5, \quad \lambda_{u}=\{0.001,0.01\}, \quad \lambda_{v}=$ $\{0.001,0.01\}$ and $\lambda=\{0.0001,0.001,0.01\}$. KerKT and all the baselines, except for PCC, were randomly initialized. The results of 20 random initializations were averaged; standard deviations are reported.
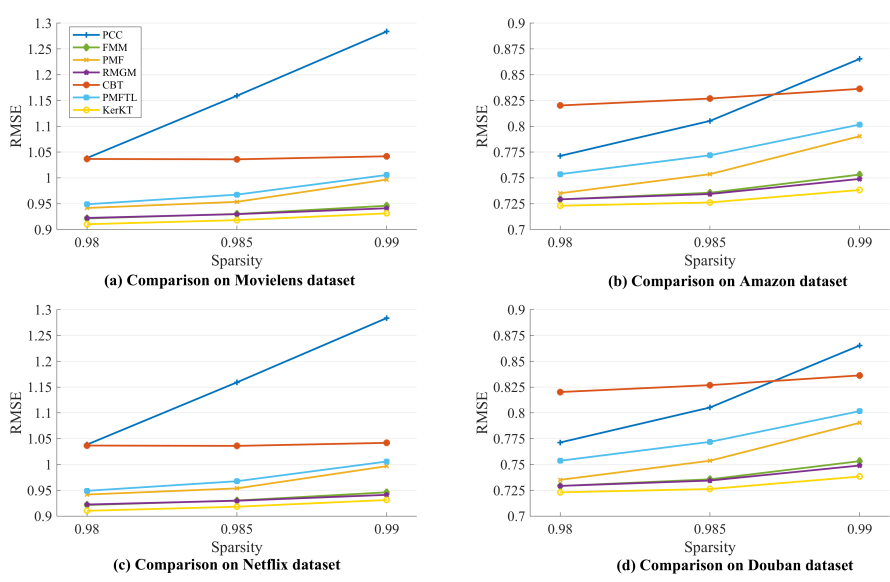

Fig. 6. Comparison results of four datasets.

\section{Results}

The results with these four datasets are shown in Tables III, IV, V and VI, and a visual comparison is shown in Fig. 6. KerKT delivered the best performance of all the comparison methods on all four cross-domain recommendation tasks. This verifies the conclusion that using overlapping entities as a bridge for transferring knowledge is useful in cross-domain recommender systems. Our analysis of the results revealed the following observations:

1) Comparison with non-transfer learning methods. The performance of non-transfer learning methods was relatively poor on sparse data. As the basis of CBT and RMGM, FMM was designed to predict ratings for users with little available data. Generally, FMM performed better than PMF and the memory-based method PCC without transfer learning techniques. PMF is the basis of our proposed method KerKT. In all the experiments, KerKT significantly outperformed all the non-transfer learning recommendation techniques.

2) Comparison with cross-domain recommendation methods for non-overlapping entities. RMGM showed improved precision in recommendations over its basis, FMM, but sometimes the improvement was not significant (see Table VI). CBT did not always improve the performance of the recommender system and sometimes suffered from negative transfer, indicating that CBT is not stable when transferring knowledge (see Table IV). Neither of these methods uses non-overlapping entity information explicitly, but rather extract clusterbased knowledge to share between the source and target domains. KerKT outperformed both these methods in all recommendation tasks, again, proving that overlapping entities can serve as a bridge for transferring knowledge to the target domain. Methods designed for scenarios with non-overlapping entities can be applied to solve the problem proposed in this paper as a substitution. But we can see from the results that they did not show advantages since they did not use the overlapping information.

3) Comparison with cross-domain recommendation 
TABLE III

OVERALL COMPARISON RESULT ON THE MOVIELENS DATA.

\begin{tabular}{|c|c|c|c|c|c|c|c|c|c|c|}
\hline & \multirow[b]{2}{*}{ Method } & \multicolumn{3}{|l|}{$1.00 \%$} & \multicolumn{3}{|l|}{$1.50 \%$} & \multicolumn{3}{|l|}{$2.00 \%$} \\
\hline & & 200 & 100 & 50 & 200 & 100 & 50 & 200 & 100 & 50 \\
\hline \multirow{7}{*}{ MAE } & $\mathrm{PCC}$ & 0.7877 & 0.7822 & 0.7825 & 0.7227 & 0.7201 & 0.7176 & 0.6923 & 0.6929 & 0.6928 \\
\hline & FMM & $\begin{array}{l}0.6708 \\
( \pm 0.0013)\end{array}$ & $\begin{array}{l}0.6727 \\
( \pm 0.0015)\end{array}$ & $\begin{array}{l}0.6704 \\
( \pm 0.0019)\end{array}$ & $\begin{array}{l}0.6527 \\
( \pm 0.0013)\end{array}$ & $\begin{array}{l}0.6532 \\
( \pm 0.0009)\end{array}$ & $\begin{array}{l}0.6496 \\
( \pm 0.0009)\end{array}$ & $\begin{array}{l}0.6458 \\
( \pm 0.0009)\end{array}$ & $\begin{array}{l}0.6471 \\
( \pm 0.0007)\end{array}$ & $\begin{array}{l}0.6498 \\
( \pm 0.0010)\end{array}$ \\
\hline & PMF & $\begin{array}{l}0.7007 \\
( \pm 0.0006)\end{array}$ & $\begin{array}{l}0.7003 \\
( \pm 0.0008)\end{array}$ & $\begin{array}{l}0.7026 \\
( \pm 0.0012)\end{array}$ & $\begin{array}{l}0.6797 \\
( \pm 0.0013)\end{array}$ & $\begin{array}{l}0.6853 \\
( \pm 0.0026)\end{array}$ & $\begin{array}{l}0.6777 \\
( \pm 0.0010)\end{array}$ & $\begin{array}{l}0.6560 \\
( \pm 0.0007)\end{array}$ & $\begin{array}{l}0.6567 \\
( \pm 0.0005)\end{array}$ & $\begin{array}{l}0.6601 \\
( \pm 0.0009)\end{array}$ \\
\hline & RMGM & $\begin{array}{l}0.6659 \\
( \pm 0.0012)\end{array}$ & $\begin{array}{l}0.6698 \\
( \pm 0.0010)\end{array}$ & $\begin{array}{l}0.6668 \\
( \pm 0.0024)\end{array}$ & $\begin{array}{l}0.6524 \\
( \pm 0.0010)\end{array}$ & $\begin{array}{l}0.6531 \\
( \pm 0.0009)\end{array}$ & $\begin{array}{l}0.6500 \\
( \pm 0.0012)\end{array}$ & $\begin{array}{l}0.6466 \\
( \pm 0.0008)\end{array}$ & $\begin{array}{l}0.6473 \\
( \pm 0.0006)\end{array}$ & $\begin{array}{l}0.6506 \\
( \pm 0.0006)\end{array}$ \\
\hline & CBT & $\begin{array}{l}0.7489 \\
( \pm 0.0030)\end{array}$ & $\begin{array}{l}0.7527 \\
( \pm 0.0017)\end{array}$ & $\begin{array}{l}0.7513 \\
( \pm 0.0020)\end{array}$ & $\begin{array}{l}0.7499 \\
( \pm 0.0041)\end{array}$ & $\begin{array}{l}0.7509 \\
( \pm 0.0037)\end{array}$ & $\begin{array}{l}0.7456 \\
( \pm 0.0030)\end{array}$ & $\begin{array}{l}0.7469 \\
( \pm 0.0029)\end{array}$ & $\begin{array}{l}0.7478 \\
( \pm 0.0033)\end{array}$ & $\begin{array}{l}0.7491 \\
( \pm 0.0029)\end{array}$ \\
\hline & PMFTL & $\begin{array}{l}0.7222 \\
( \pm 0.0023)\end{array}$ & $\begin{array}{l}0.7182 \\
( \pm 0.0017)\end{array}$ & $\begin{array}{l}0.7227 \\
( \pm 0.0008)\end{array}$ & $\begin{array}{l}0.6931 \\
( \pm 0.0042)\end{array}$ & $\begin{array}{l}0.6951 \\
( \pm 0.0050)\end{array}$ & $\begin{array}{l}0.6819 \\
( \pm 0.0047)\end{array}$ & $\begin{array}{l}0.6822 \\
( \pm 0.0036)\end{array}$ & $\begin{array}{l}0.6764 \\
( \pm 0.0015)\end{array}$ & $\begin{array}{l}0.6719 \\
( \pm 0.0037)\end{array}$ \\
\hline & KerKT & $\begin{array}{l}\mathbf{0 . 6 5 6 6} \\
( \pm 0.0029)\end{array}$ & $\begin{array}{l}\mathbf{0 . 6 5 5 3} \\
( \pm 0.0009)\end{array}$ & $\begin{array}{l}\mathbf{0 . 6 5 6 3} \\
( \pm 0.0017)\end{array}$ & $\begin{array}{l}\mathbf{0 . 6 4 1 1} \\
( \pm 0.0009)\end{array}$ & $\begin{array}{l}\mathbf{0 . 6 4 2 3} \\
( \pm 0.0005)\end{array}$ & $\begin{array}{l}\mathbf{0 . 6 4 0 5} \\
( \pm 0.0037)\end{array}$ & $\begin{array}{l}\mathbf{0 . 6 4 1 1} \\
( \pm 0.0007)\end{array}$ & $\begin{array}{l}\mathbf{0 . 6 3 7 1} \\
( \pm 0.0018)\end{array}$ & $\begin{array}{l}\mathbf{0 . 6 4 0 3} \\
( \pm 0.0005)\end{array}$ \\
\hline \multirow{7}{*}{ RMSE } & PCC & 1.0087 & 1.0028 & 1.0009 & 0.9259 & 0.9203 & 0.9172 & 0.8826 & 0.8839 & 0.8830 \\
\hline & FMM & $\begin{array}{l}0.8575 \\
( \pm 0.0014)\end{array}$ & $\begin{array}{l}0.8604 \\
( \pm 0.0017)\end{array}$ & $\begin{array}{l}0.8566 \\
( \pm 0.0019)\end{array}$ & $\begin{array}{l}0.8339 \\
( \pm 0.0016)\end{array}$ & $\begin{array}{l}0.8339 \\
( \pm 0.0010)\end{array}$ & $\begin{array}{l}0.8314 \\
( \pm 0.0012)\end{array}$ & $\begin{array}{l}0.8254 \\
( \pm 0.0010)\end{array}$ & $\begin{array}{l}0.8294 \\
( \pm 0.0010)\end{array}$ & $\begin{array}{l}0.8280 \\
( \pm 0.0011)\end{array}$ \\
\hline & PMF & $\begin{array}{l}0.8808 \\
( \pm 0.0007)\end{array}$ & $\begin{array}{l}0.8812 \\
( \pm 0.0012)\end{array}$ & $\begin{array}{l}0.8821 \\
( \pm 0.0007)\end{array}$ & $\begin{array}{l}0.8613 \\
( \pm 0.0014)\end{array}$ & $\begin{array}{l}0.8588 \\
( \pm 0.0029)\end{array}$ & $\begin{array}{l}0.8574 \\
( \pm 0.0013)\end{array}$ & $\begin{array}{l}0.8312 \\
( \pm 0.0008)\end{array}$ & $\begin{array}{l}0.8341 \\
( \pm 0.0007)\end{array}$ & $\begin{array}{l}0.8338 \\
( \pm 0.0010)\end{array}$ \\
\hline & RMGM & $\begin{array}{l}0.8509 \\
( \pm 0.0014)\end{array}$ & $\begin{array}{l}0.8563 \\
( \pm 0.0010)\end{array}$ & $\begin{array}{l}0.8511 \\
( \pm 0.0013)\end{array}$ & $\begin{array}{l}0.8327 \\
( \pm 0.0014)\end{array}$ & $\begin{array}{l}0.8330 \\
( \pm 0.0012)\end{array}$ & $\begin{array}{l}0.831 \\
( \pm 0.0011)\end{array}$ & $\begin{array}{l}0.8259 \\
( \pm 0.0009)\end{array}$ & $\begin{array}{l}0.8291 \\
( \pm 0.0007)\end{array}$ & $\begin{array}{l}0.8284 \\
( \pm 0.0010)\end{array}$ \\
\hline & CBT & $\begin{array}{l}0.9663 \\
( \pm 0.0051)\end{array}$ & $\begin{array}{l}0.9703 \\
( \pm 0.0036)\end{array}$ & $\begin{array}{l}0.9683 \\
( \pm 0.0047)\end{array}$ & $\begin{array}{l}0.9668 \\
( \pm 0.0065)\end{array}$ & $\begin{array}{l}0.9650 \\
( \pm 0.0054)\end{array}$ & $\begin{array}{l}0.9611 \\
( \pm 0.0052)\end{array}$ & $\begin{array}{l}0.9613 \\
( \pm 0.0050)\end{array}$ & $\begin{array}{l}0.9624 \\
( \pm 0.0050)\end{array}$ & $\begin{array}{l}0.9641 \\
( \pm 0.0050)\end{array}$ \\
\hline & PMFTL & $\begin{array}{l}0.9083 \\
( \pm 0.0023)\end{array}$ & $\begin{array}{l}0.9050 \\
( \pm 0.0025)\end{array}$ & $\begin{array}{l}0.9041 \\
( \pm 0.0031)\end{array}$ & $\begin{array}{l}0.8715 \\
( \pm 0.0045)\end{array}$ & $\begin{array}{l}0.8712 \\
( \pm 0.0053)\end{array}$ & $\begin{array}{l}0.8574 \\
( \pm 0.0040)\end{array}$ & $\begin{array}{l}0.8592 \\
( \pm 0.0041)\end{array}$ & $\begin{array}{l}0.8566 \\
( \pm 0.0020)\end{array}$ & $\begin{array}{l}0.8482 \\
( \pm 0.0037)\end{array}$ \\
\hline & KerKT & $\begin{array}{l}\mathbf{0 . 8 3 5 5} \\
( \pm 0.0025)\end{array}$ & $\begin{array}{l}\mathbf{0 . 8 3 5 2} \\
( \pm 0.0007)\end{array}$ & $\begin{array}{l}\mathbf{0 . 8 3 4 6} \\
( \pm 0.0013)\end{array}$ & $\begin{array}{l}\mathbf{0 . 8 1 8 0} \\
( \pm 0.0016)\end{array}$ & $\begin{array}{l}\mathbf{0 . 8 1 6 9} \\
( \pm 0.0008)\end{array}$ & $\begin{array}{l}\mathbf{0 . 8 1 8 1} \\
( \pm 0.0064)\end{array}$ & $\begin{array}{l}\mathbf{0 . 8 1 5 8} \\
( \pm 0.0008)\end{array}$ & $\begin{array}{l}\mathbf{0 . 8 1 5 6} \\
( \pm 0.0038)\end{array}$ & $\begin{array}{l}\mathbf{0 . 8 1 4 3} \\
( \pm 0.0018)\end{array}$ \\
\hline
\end{tabular}

TABLE IV

OVERALL COMPARISON RESULT ON THE NETFLIX DATA.

\begin{tabular}{|c|c|c|c|c|c|c|c|c|c|c|}
\hline & \multirow[b]{2}{*}{ Method } & \multicolumn{3}{|l|}{$1.00 \%$} & \multicolumn{3}{|l|}{$1.50 \%$} & \multicolumn{3}{|l|}{$2.00 \%$} \\
\hline & & 200 & 100 & 50 & 200 & 100 & 50 & 200 & 100 & 50 \\
\hline \multirow{7}{*}{ MAE } & PCC & 1.0191 & 1.0108 & 1.0026 & 0.9099 & 0.9079 & 0.9154 & 0.8139 & 0.8188 & 0.8224 \\
\hline & FMM & $\begin{array}{l}0.7460 \\
( \pm 0.0015)\end{array}$ & $\begin{array}{l}0.7452 \\
( \pm 0.0015)\end{array}$ & $\begin{array}{l}0.7431 \\
( \pm 0.0016)\end{array}$ & $\begin{array}{l}0.7303 \\
( \pm 0.0012)\end{array}$ & $\begin{array}{l}0.7337 \\
( \pm 0.0016)\end{array}$ & $\begin{array}{l}0.7349 \\
( \pm 0.0012)\end{array}$ & $\begin{array}{l}0.7225 \\
( \pm 0.0010)\end{array}$ & $\begin{array}{l}0.7251 \\
( \pm 0.0010)\end{array}$ & $\begin{array}{l}0.7297 \\
( \pm 0.0011)\end{array}$ \\
\hline & PMF & $\begin{array}{l}0.8121 \\
( \pm 0.0017)\end{array}$ & $\begin{array}{l}0.8119 \\
( \pm 0.0015)\end{array}$ & $\begin{array}{l}0.8111 \\
( \pm 0.0012)\end{array}$ & $\begin{array}{l}0.7681 \\
( \pm 0.0006)\end{array}$ & $\begin{array}{l}0.7729 \\
( \pm 0.0005)\end{array}$ & $\begin{array}{l}0.7750 \\
( \pm 0.0004)\end{array}$ & $\begin{array}{l}0.7474 \\
( \pm 0.0016)\end{array}$ & $\begin{array}{l}0.7514 \\
( \pm 0.0013)\end{array}$ & $\begin{array}{l}0.7567 \\
( \pm 0.0011)\end{array}$ \\
\hline & RMGM & $\begin{array}{l}0.7432 \\
( \pm 0.0012)\end{array}$ & $\begin{array}{l}0.7427 \\
( \pm 0.0010)\end{array}$ & $\begin{array}{l}0.7396 \\
( \pm 0.0014)\end{array}$ & $\begin{array}{l}0.7300 \\
( \pm 0.0010)\end{array}$ & $\begin{array}{l}0.7338 \\
( \pm 0.0008)\end{array}$ & $\begin{array}{l}0.7360 \\
( \pm 0.0015)\end{array}$ & $\begin{array}{l}0.7237 \\
( \pm 0.0009)\end{array}$ & $\begin{array}{l}0.7265 \\
( \pm 0.0010)\end{array}$ & $\begin{array}{l}0.7319 \\
( \pm 0.0010)\end{array}$ \\
\hline & CBT & $\begin{array}{l}0.8596 \\
( \pm 0.0073)\end{array}$ & $\begin{array}{l}0.8558 \\
( \pm 0.0079)\end{array}$ & $\begin{array}{l}0.8600 \\
( \pm 0.0050)\end{array}$ & $\begin{array}{l}0.8546 \\
( \pm 0.0051)\end{array}$ & $\begin{array}{l}0.8505 \\
( \pm 0.0063)\end{array}$ & $\begin{array}{l}0.8582 \\
( \pm 0.0080)\end{array}$ & $\begin{array}{l}0.8497 \\
( \pm 0.0068)\end{array}$ & $\begin{array}{l}0.8541 \\
( \pm 0.0077)\end{array}$ & $\begin{array}{l}0.8579 \\
( \pm 0.0061)\end{array}$ \\
\hline & PMFTL & $\begin{array}{l}0.8285 \\
( \pm 0.0024)\end{array}$ & $\begin{array}{l}0.8231 \\
( \pm 0.0054)\end{array}$ & $\begin{array}{l}0.8243 \\
( \pm 0.0055)\end{array}$ & $\begin{array}{l}0.7827 \\
( \pm 0.0037)\end{array}$ & $\begin{array}{l}0.7814 \\
( \pm 0.0019)\end{array}$ & $\begin{array}{l}0.7843 \\
( \pm 0.0045)\end{array}$ & $\begin{array}{l}0.7649 \\
( \pm 0.0035)\end{array}$ & $\begin{array}{l}0.7669 \\
( \pm 0.0042)\end{array}$ & $\begin{array}{l}0.7655 \\
( \pm 0.0056)\end{array}$ \\
\hline & KerKT & $\begin{array}{l}\mathbf{0 . 7 3 6 4} \\
( \pm 0.0004)\end{array}$ & $\begin{array}{l}\mathbf{0 . 7 3 7 5} \\
( \pm 0.0037)\end{array}$ & $\begin{array}{l}\mathbf{0 . 7 2 9 3} \\
( \pm 0.0014)\end{array}$ & $\begin{array}{l}\mathbf{0 . 7 1 8 3} \\
( \pm 0.0012)\end{array}$ & $\begin{array}{l}\mathbf{0 . 7 2 5 3} \\
( \pm 0.0013)\end{array}$ & $\begin{array}{l}\mathbf{0 . 7 2 5 0} \\
( \pm 0.0006)\end{array}$ & $\begin{array}{l}\mathbf{0 . 7 1 3 7} \\
( \pm 0.0016)\end{array}$ & $\begin{array}{l}\mathbf{0 . 7 1 7 2} \\
( \pm 0.0027)\end{array}$ & $\begin{array}{l}\mathbf{0 . 7 2 1 3} \\
( \pm 0.0032)\end{array}$ \\
\hline \multirow{7}{*}{ RMSE } & PCC & 1.2968 & 1.2835 & 1.2710 & 1.1571 & 1.1567 & 1.1636 & 1.0325 & 1.0384 & 1.0447 \\
\hline & FMM & $\begin{array}{l}0.9490 \\
( \pm 0.0019)\end{array}$ & $\begin{array}{l}0.9473 \\
( \pm 0.0015)\end{array}$ & $\begin{array}{l}0.9417 \\
( \pm 0.0023)\end{array}$ & $\begin{array}{l}0.9278 \\
( \pm 0.0010)\end{array}$ & $\begin{array}{l}0.9307 \\
( \pm 0.0019)\end{array}$ & $\begin{array}{l}0.9319 \\
( \pm 0.0012)\end{array}$ & $\begin{array}{l}0.9172 \\
( \pm 0.0009)\end{array}$ & $\begin{array}{l}0.9217 \\
( \pm 0.0011)\end{array}$ & $\begin{array}{l}0.9253 \\
( \pm 0.0009)\end{array}$ \\
\hline & PMF & $\begin{array}{l}0.9978 \\
( \pm 0.0016)\end{array}$ & $\begin{array}{l}0.9973 \\
( \pm 0.0013)\end{array}$ & $\begin{array}{l}0.9949 \\
( \pm 0.0013)\end{array}$ & $\begin{array}{l}0.9499 \\
( \pm 0.0007)\end{array}$ & $\begin{array}{l}0.9541 \\
( \pm 0.0005)\end{array}$ & $\begin{array}{l}0.9567 \\
( \pm 0.0005)\end{array}$ & $\begin{array}{l}0.9360 \\
( \pm 0.0018)\end{array}$ & $\begin{array}{l}0.9431 \\
( \pm 0.0018)\end{array}$ & $\begin{array}{l}0.9461 \\
( \pm 0.0014)\end{array}$ \\
\hline & RMGM & $\begin{array}{l}0.9437 \\
( \pm 0.0013)\end{array}$ & $\begin{array}{l}0.9432 \\
( \pm 0.0012)\end{array}$ & $\begin{array}{l}0.9365 \\
( \pm 0.0014)\end{array}$ & $\begin{array}{l}0.9267 \\
( \pm 0.0010)\end{array}$ & $\begin{array}{l}0.9301 \\
( \pm 0.0009)\end{array}$ & $\begin{array}{l}0.9322 \\
( \pm 0.0015)\end{array}$ & $\begin{array}{l}0.9175 \\
( \pm 0.0008)\end{array}$ & $\begin{array}{l}0.9228 \\
( \pm 0.0010)\end{array}$ & $\begin{array}{l}0.9268 \\
( \pm 0.0008)\end{array}$ \\
\hline & $\mathrm{CBT}$ & $\begin{array}{l}1.0448 \\
( \pm 0.0027)\end{array}$ & $\begin{array}{l}1.0392 \\
( \pm 0.0030)\end{array}$ & $\begin{array}{l}1.0417 \\
( \pm 0.0021)\end{array}$ & $\begin{array}{l}1.0363 \\
( \pm 0.0026)\end{array}$ & $\begin{array}{l}1.0318 \\
( \pm 0.0030)\end{array}$ & $\begin{array}{l}1.0399 \\
( \pm 0.0037)\end{array}$ & $\begin{array}{l}1.0330 \\
( \pm 0.0029)\end{array}$ & $\begin{array}{l}1.0367 \\
( \pm 0.0036)\end{array}$ & $\begin{array}{l}1.0403 \\
( \pm 0.0030)\end{array}$ \\
\hline & PMFTL & $\begin{array}{l}1.0078 \\
( \pm 0.0021)\end{array}$ & $\begin{array}{l}1.0031 \\
( \pm 0.0054)\end{array}$ & $\begin{array}{l}1.0061 \\
( \pm 0.0052)\end{array}$ & $\begin{array}{l}0.9675 \\
( \pm 0.0038)\end{array}$ & $\begin{array}{l}0.9665 \\
( \pm 0.0016)\end{array}$ & $\begin{array}{l}0.9684 \\
( \pm 0.0030)\end{array}$ & $\begin{array}{l}0.9467 \\
( \pm 0.0027)\end{array}$ & $\begin{array}{l}0.9509 \\
( \pm 0.0033)\end{array}$ & $\begin{array}{l}0.9491 \\
( \pm 0.0042)\end{array}$ \\
\hline & KerKT & $\begin{array}{l}\mathbf{0 . 9 3 4 9} \\
( \pm 0.0004)\end{array}$ & $\begin{array}{l}\mathbf{0 . 9 3 5 1} \\
( \pm 0.0032)\end{array}$ & $\begin{array}{l}\mathbf{0 . 9 2 3 6} \\
( \pm 0.0012)\end{array}$ & $\begin{array}{l}\mathbf{0 . 9 1 2 5} \\
( \pm 0.0007)\end{array}$ & $\begin{array}{l}\mathbf{0 . 9 1 9 9} \\
( \pm 0.0016)\end{array}$ & $\begin{array}{l}\mathbf{0 . 9 2 1 9} \\
( \pm 0.0008)\end{array}$ & $\begin{array}{l}\mathbf{0 . 9 0 5 7} \\
( \pm 0.0008)\end{array}$ & $\begin{array}{l}\mathbf{0 . 9 1 1 6} \\
( \pm 0.0024)\end{array}$ & $\begin{array}{l}\mathbf{0 . 9 1 3 2} \\
( \pm 0.0028)\end{array}$ \\
\hline
\end{tabular}


TABLE V

OVERALL COMPARISON RESULT ON THE AMAZONBOOK DATA.

\begin{tabular}{|c|c|c|c|c|c|c|c|c|c|c|}
\hline & \multirow[b]{2}{*}{ Method } & \multicolumn{3}{|l|}{$0.75 \%$} & \multicolumn{3}{|l|}{$0.63 \%$} & \multicolumn{3}{|l|}{$0.50 \%$} \\
\hline & & 200 & 100 & 50 & 200 & 100 & 50 & 200 & 100 & 50 \\
\hline \multirow{7}{*}{ MAE } & PCC & 0.7705 & 0.7551 & 1.0026 & 0.7704 & 0.7618 & 0.7615 & 0.7882 & 0.7718 & 0.9154 \\
\hline & FMM & $\begin{array}{l}0.6648 \\
( \pm 0.0029)\end{array}$ & $\begin{array}{l}0.6630 \\
( \pm 0.0026)\end{array}$ & $\begin{array}{l}0.7431 \\
( \pm 0.0019)\end{array}$ & $\begin{array}{l}0.6846 \\
( \pm 0.0028)\end{array}$ & $\begin{array}{l}0.6806 \\
( \pm 0.0031)\end{array}$ & $\begin{array}{l}0.6822 \\
( \pm 0.0032)\end{array}$ & $\begin{array}{l}0.7171 \\
( \pm 0.0034)\end{array}$ & $\begin{array}{l}0.7132 \\
( \pm 0.0041)\end{array}$ & $\begin{array}{l}0.7349 \\
( \pm 0.0012)\end{array}$ \\
\hline & PMF & $\begin{array}{l}0.6740 \\
( \pm 0.0011)\end{array}$ & $\begin{array}{l}0.6751 \\
( \pm 0.0004)\end{array}$ & $\begin{array}{l}0.8111 \\
( \pm 0.0012)\end{array}$ & $\begin{array}{l}0.6934 \\
( \pm 0.0003)\end{array}$ & $\begin{array}{l}0.6846 \\
( \pm 0.0010)\end{array}$ & $\begin{array}{l}0.6843 \\
( \pm 0.0006)\end{array}$ & $\begin{array}{l}0.6855 \\
( \pm 0.0001)\end{array}$ & $\begin{array}{l}0.6962 \\
( \pm 0.0001)\end{array}$ & $\begin{array}{l}0.7750 \\
( \pm 0.0004)\end{array}$ \\
\hline & RMGM & $\begin{array}{l}0.6581 \\
( \pm 0.0016)\end{array}$ & $\begin{array}{l}0.6578 \\
( \pm 0.0021)\end{array}$ & $\begin{array}{l}0.6521 \\
( \pm 0.0024)\end{array}$ & $\begin{array}{l}0.6706 \\
( \pm 0.0024)\end{array}$ & $\begin{array}{l}0.6671 \\
( \pm 0.0024)\end{array}$ & $\begin{array}{l}0.6657 \\
( \pm 0.0026)\end{array}$ & $\begin{array}{l}0.6855 \\
( \pm 0.0021)\end{array}$ & $\begin{array}{l}0.6831 \\
( \pm 0.0025)\end{array}$ & $\begin{array}{l}0.7316 \\
( \pm 0.0015)\end{array}$ \\
\hline & CBT & $\begin{array}{l}0.6677 \\
( \pm 0.0019)\end{array}$ & $\begin{array}{l}0.6678 \\
( \pm 0.0026)\end{array}$ & $\begin{array}{l}0.6717 \\
( \pm 0.0020)\end{array}$ & $\begin{array}{l}0.6734 \\
( \pm 0.0022)\end{array}$ & $\begin{array}{l}0.6712 \\
( \pm 0.0014)\end{array}$ & $\begin{array}{l}0.6714 \\
( \pm 0.0021)\end{array}$ & $\begin{array}{l}0.6753 \\
( \pm 0.0015)\end{array}$ & $\begin{array}{l}0.6719 \\
( \pm 0.0030)\end{array}$ & $\begin{array}{l}0.6731 \\
( \pm 0.0013)\end{array}$ \\
\hline & PMFTL & $\begin{array}{l}0.6759 \\
( \pm 0.0006)\end{array}$ & $\begin{array}{l}0.6796 \\
( \pm 0.0016)\end{array}$ & $\begin{array}{l}0.6815 \\
( \pm 0.0008)\end{array}$ & $\begin{array}{l}0.6855 \\
( \pm 0.0003)\end{array}$ & $\begin{array}{l}0.6863 \\
( \pm 0.0006)\end{array}$ & $\begin{array}{l}0.6844 \\
( \pm 0.0011)\end{array}$ & $\begin{array}{l}0.6886 \\
( \pm 0.0006)\end{array}$ & $\begin{array}{l}0.6882 \\
( \pm 0.0004)\end{array}$ & $\begin{array}{l}0.6902 \\
( \pm 0.0004)\end{array}$ \\
\hline & KerKT & $\begin{array}{l}\mathbf{0 . 6 5 6 2} \\
( \pm 0.0034)\end{array}$ & $\begin{array}{l}\mathbf{0 . 6 5 1 1} \\
( \pm 0.0038)\end{array}$ & $\begin{array}{l}\mathbf{0 . 6 4 2 0} \\
( \pm 0.0039)\end{array}$ & $\begin{array}{l}\mathbf{0 . 6 5 2 9} \\
( \pm 0.0030)\end{array}$ & $\begin{array}{l}\mathbf{0 . 6 5 8 0} \\
( \pm 0.0070)\end{array}$ & $\begin{array}{l}\mathbf{0 . 6 5 0 2} \\
( \pm 0.0018)\end{array}$ & $\begin{array}{l}\mathbf{0 . 6 6 0 1} \\
( \pm 0.0039)\end{array}$ & $\begin{array}{l}\text { 0.6611 } \\
( \pm 0.0061)\end{array}$ & $\begin{array}{l}\mathbf{0 . 6 6 1 1} \\
( \pm 0.0066)\end{array}$ \\
\hline \multirow{7}{*}{ RMSE } & PCC & 0.9203 & 0.9129 & 0.9949 & 0.9277 & 0.9337 & 0.9295 & 0.9462 & 0.9476 & 0.9567 \\
\hline & FMM & $\begin{array}{l}0.8732 \\
( \pm 0.0049)\end{array}$ & $\begin{array}{l}0.8668 \\
( \pm 0.0015)\end{array}$ & $\begin{array}{l}0.9417 \\
( \pm 0.0023)\end{array}$ & $\begin{array}{l}0.8951 \\
( \pm 0.0034)\end{array}$ & $\begin{array}{l}0.8916 \\
( \pm 0.0040)\end{array}$ & $\begin{array}{l}0.8956 \\
( \pm 0.0036)\end{array}$ & $\begin{array}{l}0.9402 \\
( \pm 0.0049)\end{array}$ & $\begin{array}{l}0.9361 \\
( \pm 0.0051)\end{array}$ & $\begin{array}{l}0.9319 \\
( \pm 0.0012)\end{array}$ \\
\hline & PMF & $\begin{array}{l}0.9203 \\
( \pm 0.0021)\end{array}$ & $\begin{array}{l}0.9129 \\
( \pm 0.0012)\end{array}$ & $\begin{array}{l}0.9949 \\
( \pm 0.0013)\end{array}$ & $\begin{array}{l}0.9277 \\
( \pm 0.0006)\end{array}$ & $\begin{array}{l}0.9337 \\
( \pm 0.0017)\end{array}$ & $\begin{array}{l}0.9295 \\
( \pm 0.0009)\end{array}$ & $\begin{array}{l}0.9462 \\
( \pm 0.0001)\end{array}$ & $\begin{array}{l}0.9476 \\
( \pm 0.0003)\end{array}$ & $\begin{array}{l}0.9567 \\
( \pm 0.0005)\end{array}$ \\
\hline & RMGM & $\begin{array}{l}0.8636 \\
( \pm 0.0023)\end{array}$ & $\begin{array}{l}0.8576 \\
( \pm 0.0031)\end{array}$ & $\begin{array}{l}0.8532 \\
( \pm 0.0032)\end{array}$ & $\begin{array}{l}0.8748 \\
( \pm 0.0028)\end{array}$ & $\begin{array}{l}0.8717 \\
( \pm 0.0027)\end{array}$ & $\begin{array}{l}0.8734 \\
( \pm 0.0031)\end{array}$ & $\begin{array}{l}0.8976 \\
( \pm 0.0028)\end{array}$ & $\begin{array}{l}0.8971 \\
( \pm 0.0034)\end{array}$ & $\begin{array}{l}0.9322 \\
( \pm 0.0015)\end{array}$ \\
\hline & CBT & $\begin{array}{l}0.9159 \\
( \pm 0.0031)\end{array}$ & $\begin{array}{l}0.9084 \\
( \pm 0.0041)\end{array}$ & $\begin{array}{l}0.9110 \\
( \pm 0.0034)\end{array}$ & $\begin{array}{l}0.9143 \\
( \pm 0.0031)\end{array}$ & $\begin{array}{l}0.9156 \\
( \pm 0.0024)\end{array}$ & $\begin{array}{l}0.9124 \\
( \pm 0.0030)\end{array}$ & $\begin{array}{l}0.9194 \\
( \pm 0.0032)\end{array}$ & $\begin{array}{l}0.9716 \\
( \pm 0.0042)\end{array}$ & $\begin{array}{l}0.9165 \\
( \pm 0.0020)\end{array}$ \\
\hline & PMFTL & $\begin{array}{l}0.9057 \\
( \pm 0.0016)\end{array}$ & $\begin{array}{l}0.9029 \\
( \pm 0.0016)\end{array}$ & $\begin{array}{l}0.9163 \\
( \pm 0.0012)\end{array}$ & $\begin{array}{l}0.9292 \\
( \pm 0.0005)\end{array}$ & $\begin{array}{l}0.9399 \\
( \pm 0.0012)\end{array}$ & $\begin{array}{l}0.9130 \\
( \pm 0.0019)\end{array}$ & $\begin{array}{l}0.9382 \\
( \pm 0.0013)\end{array}$ & $\begin{array}{l}0.9442 \\
( \pm 0.0006)\end{array}$ & $\begin{array}{l}0.9449 \\
( \pm 0.0003)\end{array}$ \\
\hline & KerKT & $\begin{array}{l}\mathbf{0 . 8 5 9 7} \\
( \pm 0.0049)\end{array}$ & $\begin{array}{l}\mathbf{0 . 8 4 3 0} \\
( \pm 0.0091)\end{array}$ & $\begin{array}{l}\mathbf{0 . 8 3 6 2} \\
( \pm 0.0054)\end{array}$ & $\begin{array}{l}\mathbf{0 . 8 4 8 3} \\
( \pm 0.0054)\end{array}$ & $\begin{array}{l}\mathbf{0 . 8 6 0 2} \\
( \pm 0.0137)\end{array}$ & $\begin{array}{l}\mathbf{0 . 8 4 8 5} \\
( \pm 0.0039)\end{array}$ & $\begin{array}{l}\mathbf{0 . 8 5 7 2} \\
( \pm 0.0067)\end{array}$ & $\begin{array}{l}\mathbf{0 . 8 6 4 1} \\
( \pm 0.0101)\end{array}$ & $\begin{array}{l}\mathbf{0 . 8 5 7 2} \\
( \pm 0.0104)\end{array}$ \\
\hline
\end{tabular}

TABLE VI

OVERALL COMPARISON RESULT ON THE DOUBAN DATA.

\begin{tabular}{|c|c|c|c|c|c|c|c|c|c|c|}
\hline & \multirow[b]{2}{*}{ Method } & \multicolumn{3}{|l|}{$1.00 \%$} & \multicolumn{3}{|l|}{$1.50 \%$} & \multicolumn{3}{|l|}{$2.00 \%$} \\
\hline & & 200 & 100 & 50 & 200 & 100 & 50 & 200 & 100 & 50 \\
\hline \multirow{7}{*}{ MAE } & PCC & 0.6695 & 0.6700 & 0.6700 & 0.6317 & 0.6306 & 0.6298 & 0.6076 & 0.6079 & 0.6154 \\
\hline & FMM & $\begin{array}{l}0.5950 \\
( \pm 0.0011)\end{array}$ & $\begin{array}{l}0.6013 \\
( \pm 0.0015)\end{array}$ & $\begin{array}{l}0.5936 \\
( \pm 0.0009)\end{array}$ & $\begin{array}{l}0.5834 \\
( \pm 0.0008)\end{array}$ & $\begin{array}{l}0.5857 \\
( \pm 0.0011)\end{array}$ & $\begin{array}{l}0.5834 \\
( \pm 0.0006)\end{array}$ & $\begin{array}{l}0.5775 \\
( \pm 0.0008)\end{array}$ & $\begin{array}{l}0.5828 \\
( \pm 0.0010)\end{array}$ & $\begin{array}{l}0.5787 \\
( \pm 0.0008)\end{array}$ \\
\hline & PMF & $\begin{array}{l}0.6256 \\
( \pm 0.0008)\end{array}$ & $\begin{array}{l}0.6288 \\
( \pm 0.0008)\end{array}$ & $\begin{array}{l}0.6248 \\
( \pm 0.0011)\end{array}$ & $\begin{array}{l}0.5962 \\
( \pm 0.0008)\end{array}$ & $\begin{array}{l}0.6033 \\
( \pm 0.0011)\end{array}$ & $\begin{array}{l}0.5993 \\
( \pm 0.0011)\end{array}$ & $\begin{array}{l}0.5848 \\
( \pm 0.0004)\end{array}$ & $\begin{array}{l}0.5864 \\
( \pm 0.0005)\end{array}$ & $\begin{array}{l}0.5867 \\
( \pm 0.0003)\end{array}$ \\
\hline & RMGM & $\begin{array}{l}0.5929 \\
( \pm 0.0008)\end{array}$ & $\begin{array}{l}0.6004 \\
( \pm 0.0013)\end{array}$ & $\begin{array}{l}0.5912 \\
( \pm 0.0011)\end{array}$ & $\begin{array}{l}0.5835 \\
( \pm 0.0009)\end{array}$ & $\begin{array}{l}0.5860 \\
( \pm 0.0009)\end{array}$ & $\begin{array}{l}0.5832 \\
( \pm 0.0005)\end{array}$ & $\begin{array}{l}0.5773 \\
( \pm 0.0010)\end{array}$ & $\begin{array}{l}0.5835 \\
( \pm 0.0011)\end{array}$ & $\begin{array}{l}0.5795 \\
( \pm 0.0006)\end{array}$ \\
\hline & CBT & $\begin{array}{l}0.6422 \\
( \pm 0.0138)\end{array}$ & $\begin{array}{l}0.6627 \\
( \pm 0.0126)\end{array}$ & $\begin{array}{l}0.6453 \\
( \pm 0.0145)\end{array}$ & $\begin{array}{l}0.6410 \\
( \pm 0.0149)\end{array}$ & $\begin{array}{l}0.6524 \\
( \pm 0.0114)\end{array}$ & $\begin{array}{l}0.6324 \\
( \pm 0.0135)\end{array}$ & $\begin{array}{l}0.6346 \\
( \pm 0.0096)\end{array}$ & $\begin{array}{l}0.6418 \\
( \pm 0.0109)\end{array}$ & $\begin{array}{l}0.6373 \\
( \pm 0.0115)\end{array}$ \\
\hline & PMFTL & $\begin{array}{l}0.6308 \\
( \pm 0.0034)\end{array}$ & $\begin{array}{l}0.6353 \\
( \pm 0.0009)\end{array}$ & $\begin{array}{l}0.6256 \\
( \pm 0.0011)\end{array}$ & $\begin{array}{l}0.6101 \\
( \pm 0.0009)\end{array}$ & $\begin{array}{l}0.6139 \\
( \pm 0.0033)\end{array}$ & $\begin{array}{l}0.6077 \\
( \pm 0.0032)\end{array}$ & $\begin{array}{l}0.6009 \\
( \pm 0.0019)\end{array}$ & $\begin{array}{l}0.5984 \\
( \pm 0.0058)\end{array}$ & $\begin{array}{l}0.5949 \\
( \pm 0.0015)\end{array}$ \\
\hline & KerKT & $\begin{array}{l}\mathbf{0 . 5 8 6 3} \\
( \pm 0.0013)\end{array}$ & $\begin{array}{l}\mathbf{0 . 5 9 2 2} \\
( \pm 0.0020)\end{array}$ & $\begin{array}{l}\mathbf{0 . 5 8 3 5} \\
( \pm 0.0012)\end{array}$ & $\begin{array}{l}\mathbf{0 . 5 7 7 8} \\
( \pm 0.0013)\end{array}$ & $\begin{array}{l}\mathbf{0 . 5 8 1 2} \\
( \pm 0.0020)\end{array}$ & $\begin{array}{l}\mathbf{0 . 5 7 8 8} \\
( \pm 0.0019)\end{array}$ & $\begin{array}{l}\mathbf{0 . 5 7 2 1} \\
( \pm 0.0005)\end{array}$ & $\begin{array}{l}\mathbf{0 . 5 7 5 2} \\
( \pm 0.0016)\end{array}$ & $\begin{array}{l}\mathbf{0 . 5 7 5 7} \\
( \pm 0.0011)\end{array}$ \\
\hline \multirow{7}{*}{ RMSE } & PCC & 0.8618 & 0.8686 & 0.8655 & 0.8052 & 0.8082 & 0.8021 & 0.7672 & 0.7724 & 0.7744 \\
\hline & FMM & $\begin{array}{l}0.7508 \\
( \pm 0.0014)\end{array}$ & $\begin{array}{l}0.7585 \\
( \pm 0.0020)\end{array}$ & $\begin{array}{l}0.7504 \\
( \pm 0.0011)\end{array}$ & $\begin{array}{l}0.7358 \\
( \pm 0.0007)\end{array}$ & $\begin{array}{l}0.7373 \\
( \pm 0.0012)\end{array}$ & $\begin{array}{l}0.7337 \\
( \pm 0.0006)\end{array}$ & $\begin{array}{l}0.7267 \\
( \pm 0.0007)\end{array}$ & $\begin{array}{l}0.7332 \\
( \pm 0.0012)\end{array}$ & $\begin{array}{l}0.7278 \\
( \pm 0.0008)\end{array}$ \\
\hline & PMF & $\begin{array}{l}0.7876 \\
( \pm 0.0011)\end{array}$ & $\begin{array}{l}0.7980 \\
( \pm 0.0015)\end{array}$ & $\begin{array}{l}0.7857 \\
( \pm 0.0015)\end{array}$ & $\begin{array}{l}0.7500 \\
( \pm 0.0009)\end{array}$ & $\begin{array}{l}0.7609 \\
( \pm 0.0014)\end{array}$ & $\begin{array}{l}0.7498 \\
( \pm 0.0013)\end{array}$ & $\begin{array}{l}0.7327 \\
( \pm 0.0005)\end{array}$ & $\begin{array}{l}0.7394 \\
( \pm 0.0009)\end{array}$ & $\begin{array}{l}0.7332 \\
( \pm 0.0003)\end{array}$ \\
\hline & RMGM & $\begin{array}{l}0.7461 \\
( \pm 0.0009)\end{array}$ & $\begin{array}{l}0.7557 \\
( \pm 0.0016)\end{array}$ & $\begin{array}{l}0.7452 \\
( \pm 0.0010)\end{array}$ & $\begin{array}{l}0.7344 \\
( \pm 0.0006)\end{array}$ & $\begin{array}{l}0.7371 \\
( \pm 0.0012)\end{array}$ & $\begin{array}{l}0.7316 \\
( \pm 0.0007)\end{array}$ & $\begin{array}{l}0.7261 \\
( \pm 0.0008)\end{array}$ & $\begin{array}{l}0.7341 \\
( \pm 0.0015)\end{array}$ & $\begin{array}{l}0.7273 \\
( \pm 0.0007)\end{array}$ \\
\hline & $\mathrm{CBT}$ & $\begin{array}{l}0.8292 \\
( \pm 0.0054)\end{array}$ & $\begin{array}{l}0.8484 \\
( \pm 0.0050)\end{array}$ & $\begin{array}{l}0.8312 \\
( \pm 0.0074)\end{array}$ & $\begin{array}{l}0.8239 \\
( \pm 0.0071)\end{array}$ & $\begin{array}{l}0.8378 \\
( \pm 0.0057)\end{array}$ & $\begin{array}{l}0.8189 \\
( \pm 0.0083)\end{array}$ & $\begin{array}{l}0.8159 \\
( \pm 0.0041)\end{array}$ & $\begin{array}{l}0.8290 \\
( \pm 0.0041)\end{array}$ & $\begin{array}{l}0.8158 \\
( \pm 0.0054)\end{array}$ \\
\hline & PMFTL & $\begin{array}{l}0.8059 \\
( \pm 0.0044)\end{array}$ & $\begin{array}{l}0.8095 \\
( \pm 0.0017)\end{array}$ & $\begin{array}{l}0.7896 \\
( \pm 0.0013)\end{array}$ & $\begin{array}{l}0.7761 \\
( \pm 0.0017)\end{array}$ & $\begin{array}{l}0.7761 \\
( \pm 0.0046)\end{array}$ & $\begin{array}{l}0.7635 \\
( \pm 0.0046)\end{array}$ & $\begin{array}{l}0.7605 \\
( \pm 0.0033)\end{array}$ & $\begin{array}{l}0.7548 \\
( \pm 0.0085)\end{array}$ & $\begin{array}{l}0.7454 \\
( \pm 0.0018)\end{array}$ \\
\hline & KerKT & $\begin{array}{l}\mathbf{0 . 7 3 6 9} \\
( \pm 0.0013)\end{array}$ & $\begin{array}{l}\mathbf{0 . 7 4 2 9} \\
( \pm 0.0027)\end{array}$ & $\begin{array}{l}\mathbf{0 . 7 3 5 2} \\
( \pm 0.0013)\end{array}$ & $\begin{array}{l}\mathbf{0 . 7 2 6 8} \\
( \pm 0.0008)\end{array}$ & $\begin{array}{l}\mathbf{0 . 7 2 7 2} \\
( \pm 0.0024)\end{array}$ & $\begin{array}{l}\mathbf{0 . 7 2 4 6} \\
( \pm 0.0022)\end{array}$ & $\begin{array}{l}\mathbf{0 . 7 1 8 5} \\
( \pm 0.0007)\end{array}$ & $\begin{array}{l}\mathbf{0 . 7 2 5 8} \\
( \pm 0.0036)\end{array}$ & $\begin{array}{l}\mathbf{0 . 7 2 4 8} \\
( \pm 0.0023)\end{array}$ \\
\hline
\end{tabular}


TABLE VII

OVERALL AVERAGE RESULT ON FOUR TASKS.

\begin{tabular}{lllllllllll}
\hline & & \multicolumn{3}{l}{ Non-transfer } & & & \multicolumn{2}{l}{ Cross-domain } \\
\cline { 3 - 4 } & & PCC & PMF & FMM & & CBT & PMFTL & RMGM & KerKT \\
\hline \multirow{2}{*}{ MAE } & Task1 & 0.9134 & 0.7773 & 0.7338 & & 0.8541 & 0.7913 & 0.7333 & $\mathbf{0 . 7 2 4 7}$ \\
& Task2 & 0.7696 & 0.6848 & 0.6872 & & 0.6712 & 0.6840 & 0.6704 & $\mathbf{0 . 6 5 6 6}$ \\
& Task3 & 0.7330 & 0.6798 & 0.6571 & & 0.7495 & 0.6979 & 0.6559 & $\mathbf{0 . 6 4 5 6}$ \\
& Task4 & 0.6369 & 0.6040 & 0.5868 & & 0.6433 & 0.6131 & 0.5864 & $\mathbf{0 . 5 8 0 3}$ \\
& & & & & & & & & \\
RMSE & Task1 & 1.1608 & 0.9630 & 0.9323 & & 1.0370 & 0.9738 & 0.9307 & $\mathbf{0 . 9 2 0 0}$ \\
& Task2 & 0.9314 & 0.9314 & 0.9005 & & 0.9242 & 0.9267 & 0.8771 & $\mathbf{0 . 8 5 5 4}$ \\
& Task3 & 0.9374 & 0.8579 & 0.8401 & & 0.9654 & 0.8786 & 0.8380 & $\mathbf{0 . 8 2 2 8}$ \\
& Task4 & 0.8139 & 0.7597 & 0.7394 & & 0.8278 & 0.7757 & 0.7375 & $\mathbf{0 . 7 2 9 2}$ \\
\hline
\end{tabular}

TABLE VIII

OVERALL IMPROVEMENT OF KERKT ON FOUR TASKS.

\begin{tabular}{|c|c|c|c|c|c|c|c|}
\hline & & \multicolumn{3}{|c|}{ Non-transfer } & \multicolumn{3}{|c|}{ Cross-domain } \\
\hline & & PCC & PMF & FMM & CBT & PMFTL & RMGM \\
\hline \multirow[t]{4}{*}{ MAE } & Task1 & $20.66 \%$ & $6.76 \%$ & $1.24 \%$ & $15.14 \%$ & $8.41 \%$ & $1.17 \%$ \\
\hline & Task2 & $14.69 \%$ & $4.12 \%$ & $4.46 \%$ & $2.18 \%$ & $4.01 \%$ & $2.06 \%$ \\
\hline & Task3 & $11.92 \%$ & $5.03 \%$ & $1.75 \%$ & $13.87 \%$ & $7.49 \%$ & $1.57 \%$ \\
\hline & Task4 & $8.89 \%$ & $3.92 \%$ & $1.11 \%$ & $9.79 \%$ & $5.34 \%$ & $1.04 \%$ \\
\hline \multirow[t]{4}{*}{ RMSE } & Task1 & $20.75 \%$ & $4.47 \%$ & $1.32 \%$ & $11.28 \%$ & $5.53 \%$ & $1.15 \%$ \\
\hline & Task2 & $8.16 \%$ & $8.16 \%$ & $5.01 \%$ & $7.44 \%$ & $7.69 \%$ & $2.47 \%$ \\
\hline & Task3 & $12.22 \%$ & $4.09 \%$ & $2.05 \%$ & $14.76 \%$ & $6.35 \%$ & $1.81 \%$ \\
\hline & Task4 & $10.41 \%$ & $4.02 \%$ & $1.38 \%$ & $11.91 \%$ & $6.00 \%$ & $1.13 \%$ \\
\hline
\end{tabular}

methods for partially overlapping entities. As methods designed for partially overlapping entities are rare and methods developed for fully overlapping entities cannot be used to solve the problem proposed in this paper, only PMFTL serves as a representative of crossdomain recommendation method for partially overlapping entities. PMFTL was developed on the basis of PMF with partially overlapping entities. The results of the experiments show that PMFTL was not effective in every situation since it ignores divergence between the source and target domains. As a result, KerKT outperformed PMFTL in each of the four tasks with all three data sparsities.

4) The number of overlapping entities. We tested three different levels of overlapping entities in these experiments: 200, 100, and 50. There were 2000 users/items in the target domain, so these proportions of overlapping entities represent just a small number of the overall total. However, even these small proportions still allowed knowledge to be transferred from the source to the target domain. We did not observe a very obvious increase/decrease in the precision of KerKT as the number of overlapping entities increased.

To further study the overall effectiveness of the KerKT method on these four tasks, we calculated the average MAEs and RMSEs as displayed in Table VII. The results show that in each task, KerKT outperformed all the other baselines. Table VIII contains the percentage of improvement, which shows that KerKT delivered the greatest improvement over the memory-based recommendation method PCC by around $20 \%$. Compared to the cross-domain recommendation method RMGM, KerKT attained an improvement of $2 \%$. It again shows that our method has the advantage when transferring knowledge from the source domain to the target domain.
TABLE IX

ELAPSED TIME COMPARISON ON NETFLIX DATASET

\begin{tabular}{|c|c|c|c|c|c|c|c|}
\hline \multirow[b]{2}{*}{ Method } & \multicolumn{3}{|c|}{ Non-transfer } & \multicolumn{4}{|c|}{ Cross-domain } \\
\hline & PCC & PMF & FMM & CBT & PMFTL & RMGM & KerKT \\
\hline Time(s) & 12.32 & 11.31 & 5.92 & 525.95 & 65.99 & 220.43 & 188.33 \\
\hline
\end{tabular}

\section{Complexity Analysis}

This complexity analysis covers each step of the proposed method KerKT. For simplicity, the dimensions of the user and item features in both domains have all been set to $k$. The time complexity of each step is listed below.

1) Step 1

a) - $O\left(k n^{2}\right)$, for each iteration to update $\boldsymbol{U}_{s}^{(0)}, \boldsymbol{V}_{s}^{(0)}$, $\boldsymbol{U}_{t}^{(0)}$ and $\boldsymbol{V}_{t}^{(0)}$, and for each iteration to approximate $\boldsymbol{X}_{s}$ and $\boldsymbol{X}_{t}$ for the matrix factorization.

b) - $O\left(k^{2} n\right)$, for the domain adaptation process to adjust the feature matrixes of the overlapped entities.

2) Step $2-O\left(k n^{2}\right)$ for each iteration to update the feature matrixes without the overlapped entities.

3) Step $3-O\left(k n^{2}\right)$ to calculate the similarity matrix of users and items.

4) Step $4-O\left(n^{3}\right)$ for the diffusion kernel completion.

5) Step $5-O\left(k n^{2}\right)$ for each iteration to update Eq. (22).

Since the number of iterations in Steps 3 and 5 are not infinite, KerKT's the total complexity is $O\left(n^{3}\right)$. We have also listed the time taken for each of the methods to complete Task 2 in Table IX. This experiment was conducted with 200 overlapping entities and $0.5 \%$ sparsity on a computer with 16GB memory and $2.2 \mathrm{GHz}$ Intel Core i7. We can see that the non-transfer methods were faster. This is due to their general simplicity and because they do not take cross-domain data into consideration. Most of the time was spent on the user and item similarity matrix calculations. However, some parallel computing could be used to speed up these computations. Alternatively, these matrixes could be pre-calculated and stored so as not to affect the speed of online recommendation. Overall, the time complexity analysis shows that the KerKT method can be used with large-scale datasets and for online e-commerce or business-to-business systems.

\section{E. Parameter Analysis}

There are three important parameters in KerKT: $\lambda_{u}, \lambda_{v}$ and $\lambda$. Each is a trade-off parameter in Equation (21). For simplicity, we have only presented the results for the Movielens dataset. This experiment was conducted with a sparsity ratio of $99.0 \%$ and 200 overlapping entities. $M A E$ and $R M S E$ were used as metrics. The results are presented in Figures 7 and 8.

To analyze the parameters $\lambda_{u}$ and $\lambda_{v}$, we set parameter $\lambda$ to 0.0001. From Figure 7, we can see that the $M A E$ and $R M S E$ change with different settings for $\lambda_{u}$ and $\lambda_{v}$. These parameters reflect the influence of the user and item similarities on the matrix factorization while parameter $\lambda$ restricts the complexity of the algorithm to avoid overfitting. We used a grid search to find the optimized settings for each of these parameters, $\lambda$, $\lambda_{u}$ and $\lambda_{v}$, which resulted in a setting of 0.01 for all. 

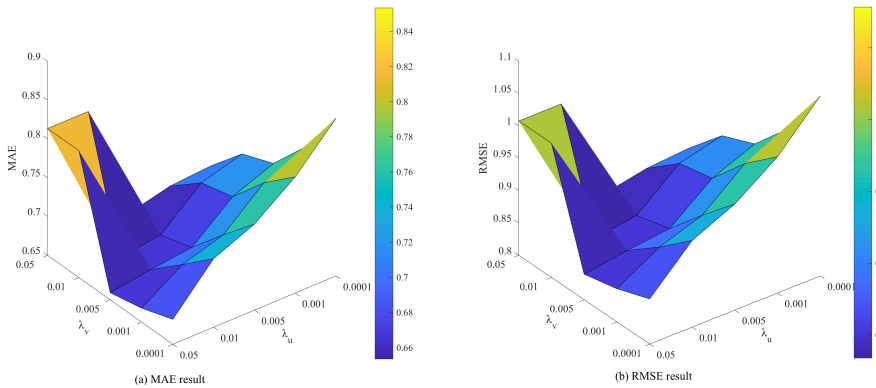

Fig. 7. Result of Movielens dataset with different $\lambda_{u}, \lambda_{v}$ parameter settings.
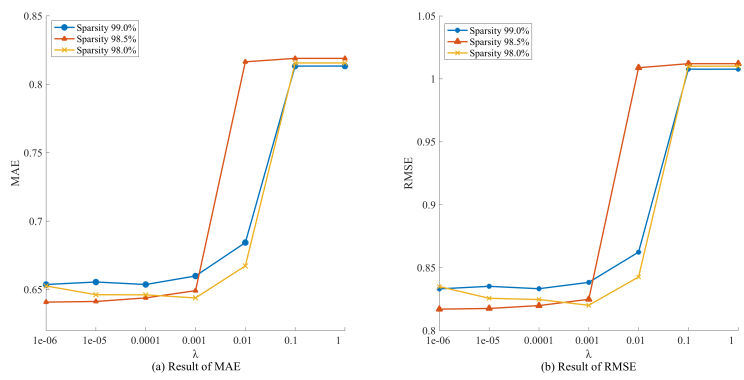

Fig. 8. Result of Movielens dataset with different $\lambda$ parameter settings.

\section{CONCLUSION AND Further Study}

Today's landscape of online sales is characterized by many websites, all selling the same item, and many online shoppers with a multitude of shopping choices. Hence, in practice, it is quite common for cross-domain recommender systems to encounter overlapping entities. This paper presents a novel cross-domain recommendation method for knowledge transfer, called KerKT. This method exploits overlapping entities as a bridge between the source and target domains and is applicable to e-commerce websites, such as Amazon, where book rating data are very dense but data in other categories are sparse. Unlike previous research, KerKT does not require that the entities be fully overlapped; it performs well in scenarios with partially overlapping entities. One advantage of this method is that it aligns the latent features of the entities extracted from the original ratings matrix. This fixes shifts in the entity feature space caused by user preference deviations between the domains. Further, the entity similarity matrix is completed through diffusion kernel completion to tackle the inconsistencies caused by heterogeneous feature spaces between two domains. The similarity matrix is extended into matrix factorization with more flexible constraints to integrate the overlapping entity information. Experimental results from a comparison with six non-transfer learning and cross-domain recommendation methods show that KerKT achieved the best performance. Even with a small ratio of overlapping entities, it was still possible to transfer knowledge from the source domain to the target domain.

There is practical significance in studying and developing cross-domain recommender systems. Smart BizSeeker, a B2B recommender system, aims to recommend appropriate business partners to businesses in Australia [43]. In a future study, we plan to implementing our proposed method into their system. Furthermore, there are still some interesting issues to be explored. For example, how to choose the best source domain if several domains are available? And which sparsity ratio in the target domain benefits transfer learning the most? In future work, we intend to apply our method to other kinds of data beyond ratings, such as web browser records and social media records. Methods for integrating explicit and implicit data within one system and extracting common knowledge from two domains with different data structures are still open and challenging issues.

\section{ACKNOWLEDGMENTS}

This work was supported by the Australian Research Council (ARC) under Discovery Grant [DP170101632]. We thank Feng Liu for his valuable discussions on transfer learning and Jemima Moore for her proofreading of this paper.

\section{REFERENCES}

[1] J. Lu, D. Wu, M. Mao, W. Wang, and G. Zhang, "Recommender system application developments: a survey," Decision Support Systems, vol. 74, pp. 12-32, 2015.

[2] X. Luo, M. Zhou, S. Li, Z. You, Y. Xia, and Q. Zhu, "A nonnegative latent factor model for large-scale sparse matrices in recommender systems via alternating direction method," IEEE Transactions on Neural Networks and Learning Systems, vol. 27, no. 3, pp. 579-592, 2016.

[3] S. Boutemedjet and D. Ziou, "Predictive approach for user long-term needs in content-based image suggestion," IEEE Transactions on Neural Networks and Learning Systems, vol. 23, no. 8, pp. 1242-1253, 2012.

[4] F. Zhang, N. J. Yuan, D. Lian, X. Xie, and W.-Y. Ma, "Collaborative knowledge base embedding for recommender systems," in Proceedings of the 22nd ACM SIGKDD International Conference on Knowledge Discovery and Data Mining. ACM, 2016, pp. 353-362.

[5] Y. Koren, "Factorization meets the neighborhood: a multifaceted collaborative filtering model," in Proceedings of the 14th ACM SIGKDD International Conference on Knowledge Discovery and Data Mining. ACM, 2008, pp. 426-434.

[6] N. Guan, D. Tao, Z. Luo, and B. Yuan, "Online nonnegative matrix factorization with robust stochastic approximation," IEEE Transactions on Neural Networks and Learning Systems, vol. 23, no. 7, pp. 10871099, 2012.

[7] B. Liu, H. Xiong, S. Papadimitriou, Y. Fu, and Z. Yao, "A general geographical probabilistic factor model for point of interest recommendation," IEEE Transactions on Knowledge and Data Engineering, vol. 27, no. 5, pp. 1167-1179, 2015.

[8] H. Wang, N. Wang, and D.-Y. Yeung, "Collaborative deep learning for recommender systems," in Proceedings of the 21th ACM SIGKDD International Conference on Knowledge Discovery and Data Mining. ACM, 2015, pp. 1235-1244.

[9] A. Stuhlsatz, J. Lippel, and T. Zielke, "Feature extraction with deep neural networks by a generalized discriminant analysis," IEEE Transactions on Neural Networks and Learning Systems, vol. 23, no. 4, pp. 596-608, 2012.

[10] J. Lu, J. Xuan, G. Zhang, and X. Luo, "Structural property-aware multilayer network embedding for latent factor analysis," Pattern Recognition, vol. 76, pp. 228-241, 2018.

[11] J.-D. Zhang, C.-Y. Chow, and J. Xu, "Enabling kernel-based attributeaware matrix factorization for rating prediction," IEEE Transactions on Knowledge and Data Engineering, vol. 29, no. 4, pp. 798-812, 2017.

[12] S. Deng, L. Huang, G. Xu, X. Wu, and Z. Wu, "On deep learning for trust-aware recommendations in social networks," IEEE Transactions on Neural Networks and Learning Systems, vol. 28, no. 5, pp. 1164-1177, 2017.

[13] B. Yang, Y. Lei, J. Liu, and W. Li, "Social collaborative filtering by trust," IEEE Transactions on Pattern Analysis and Machine Intelligence, vol. 39, no. 8, pp. 1633-1647, 2017.

[14] M. Long, J. Wang, G. Ding, S. J. Pan, and S. Y. Philip, "Adaptation regularization: A general framework for transfer learning," IEEE Transactions on Knowledge and Data Engineering, vol. 26, no. 5, pp. 1076$1089,2014$. 
[15] J. Liu, Y. Jiang, Z. Li, X. Zhang, and H. Lu, "Domain-sensitive recommendation with user-item subgroup analysis," IEEE Transactions on Knowledge and Data Engineering, vol. 28, no. 4, pp. 939-950, 2016.

[16] S. Rendle and L. Schmidt-Thieme, "Online-updating regularized kernel matrix factorization models for large-scale recommender systems," in Proceedings of the 2008 ACM Conference on Recommender Systems. ACM, 2008, pp. 251-258.

[17] N. D. Lawrence and R. Urtasun, "Non-linear matrix factorization with gaussian processes," in Proceedings of the 26th Annual International Conference on Machine Learning. ACM, 2009, pp. 601-608.

[18] M. A. Ghazanfar, A. Prügel-Bennett, and S. Szedmak, "Kernel-mapping recommender system algorithms," Information Sciences, vol. 208, pp. 81-104, 2012

[19] R. R. Coifman and S. Lafon, "Diffusion maps," Applied and computational harmonic analysis, vol. 21, no. 1, pp. 5-30, 2006.

[20] R. I. Kondor and J. Lafferty, "Diffusion kernels on graphs and other discrete input spaces," in ICML, vol. 2, 2002, pp. 315-322.

[21] A. P. Singh and G. J. Gordon, "Relational learning via collective matrix factorization," in Proceedings of the 14th ACM SIGKDD International Conference on Knowledge Discovery and Data Mining. ACM, 2008, pp. 650-658.

[22] Y. Zhen, W.-J. Li, and D.-Y. Yeung, "Tagicofi: tag informed collaborative filtering," in Proceedings of the 3rd ACM Conference on Recommender Systems. ACM, 2009, pp. 69-76.

[23] P. Hao, G. Zhang, L. Martinez, and J. Lu, "Regularizing knowledge transfer in recommendation with tag-inferred correlation," IEEE Transactions on Cybernetics, 2017.

[24] M. Jiang, P. Cui, X. Chen, F. Wang, W. Zhu, and S. Yang, "Social recommendation with cross-domain transferable knowledge," IEEE Transactions on Knowledge and Data Engineering, vol. 27, no. 11, pp. 3084-3097, 2015.

[25] M. Pratama, J. Lu, G. Zhang et al., "Evolving type-2 fuzzy classifier," IEEE Transaction on Fuzzy Systems, vol. 24, no. 3, pp. 574-589, 2016.

[26] B. Li, Q. Yang, and X. Xue, "Can movies and books collaborate? crossdomain collaborative filtering for sparsity reduction." in IJCAI, vol. 9, 2009, pp. 2052-2057.

[27] _ , "Transfer learning for collaborative filtering via a rating-matrix generative model," in Proceedings of the 26th annual international conference on machine learning. ACM, 2009, pp. 617-624.

[28] Q. Zhang, D. Wu, J. Lu, F. Liu, and G. Zhang, "A cross-domain recommender system with consistent information transfer," Decision Support Systems, vol. 104, pp. 49-63, 2017.

[29] W. Pan and Q. Yang, "Transfer learning in heterogeneous collaborative filtering domains," Artificial intelligence, vol. 197, pp. 39-55, 2013

[30] L. Hu, J. Cao, G. Xu, L. Cao, Z. Gu, and C. Zhu, "Personalized recommendation via cross-domain triadic factorization," in Proceedings of the 22nd International Conference on World Wide Web. ACM, 2013, pp. 595-606.

[31] N. Mirbakhsh and C. X. Ling, "Improving top-n recommendation for cold-start users via cross-domain information," ACM Transactions on Knowledge Discovery from Data, vol. 9, no. 4, p. 33, 2015.

[32] C.-Y. Li and S.-D. Lin, "Matching users and items across domains to improve the recommendation quality," in Proceedings of the 20th ACM SIGKDD International Conference on Knowledge Discovery and Data Mining. ACM, 2014, pp. 801-810.

[33] L. Zhao, S. J. Pan, and Q. Yang, "A unified framework of active transfer learning for cross-system recommendation," Artificial Intelligence, vol. 245, pp. 38-55, 2017.

[34] Y. Koren, R. Bell, and C. Volinsky, "Matrix factorization techniques for recommender systems," Computer, vol. 42, no. 8, pp. 30-37, 2009.

[35] A. Mnih and R. R. Salakhutdinov, "Probabilistic matrix factorization," in Advances in Neural Information Processing Systems, 2008, pp. 12571264.

[36] B. Gong, K. Grauman, and F. Sha, "Learning kernels for unsupervised domain adaptation with applications to visual object recognition," International Journal of Computer Vision, vol. 109, no. 1-2, pp. 3-27, 2014.

[37] C. H. Nguyen and H. Mamitsuka, "Latent feature kernels for link prediction on sparse graphs," IEEE Transactions on Neural Networks and Learning Systems, vol. 23, no. 11, pp. 1793-1804, 2012.

[38] W.-C. Chang, Y. Wu, H. Liu, and Y. Yang, "Cross-domain kernel induction for transfer learning." in AAAI, 2017, pp. 1763-1769.

[39] R. He and J. McAuley, "Ups and downs: Modeling the visual evolution of fashion trends with one-class collaborative filtering," in Proceedings of the 25th International Conference on World Wide Web. International World Wide Web Conferences Steering Committee, 2016, pp. 507-517.
[40] E. Zhong, W. Fan, and Q. Yang, "User behavior learning and transfer in composite social networks," ACM Transactions on Knowledge Discovery from Data, vol. 8, no. 1, pp. 6:1-6:32, Feb. 2014.

[41] M. Deshpande and G. Karypis, "Item-based top-n recommendation algorithms," ACM Transactions on Information Systems, vol. 22, no. 1, pp. 143-177, 2004.

[42] L. Si and R. Jin, "Flexible mixture model for collaborative filtering," in Proceedings of the 20th International Conference on Machine Learning, 2003, pp. 704-711.

[43] D. Wu, G. Zhang, and J. Lu, "A fuzzy preference tree-based recommender system for personalized business-to-business e-services," IEEE Transactions on Fuzzy Systems, vol. 23, no. 1, pp. 29-43, 2015.

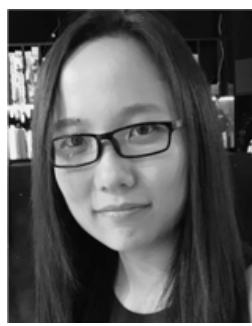

Qian Zhang is a PhD student and a member of the Decision Systems and e-Service Intelligent (DeSI) Research Lab at the Center for Artificial Intelligence, Faculty of Engineering and Information Technology, University of Technology Sydney, Australia. Her research interests include recommender systems and personalized techniques. She specializes in crossdomain recommender systems.

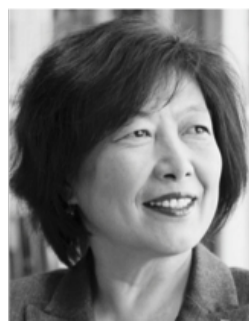

Jie Lu is a Distinguished Professor, the Director of the Center for Artificial Intelligence, and the Associate Dean (Research Excellence) with the Faculty of Engineering and Information Technology at the University of Technology Sydney, Australia. She received her $\mathrm{PhD}$ in information systems from the Curtin University of Technology, Australia, in 2000. Her research expertise spans fuzzy transfer learning, decision support systems, recommender systems and concept drift. She has published 10 research books and over 400 papers in refereed journals and conference proceedings, with over 170 papers in IEEE Transactions and other international journals. She has received over 20 Australian Research Council (ARC) Discovery Project grants and many other research projects. She serves as Editor-In-Chief for Knowledge-Based Systems (Elsevier), Editor-In-Chief for the International Journal on Computational Intelligence Systems (Atlantis), Associate Editor for IEEE Transactions on Fuzzy Systems, and has served as a guest editor of 12 special issues, general/PC/organization chairs for ten international conferences as well as having delivered 20 keynote/plenary speeches at IEEE and other international conferences. She is a Fellow of IEEE and Fellow of IFSA.

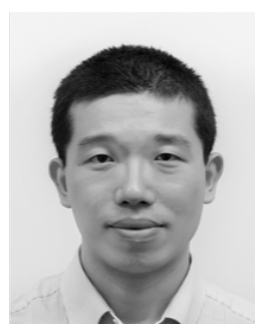

Dianshuang Wu is a Postdoctoral Research Fellow and a member of the Decision Systems and e-Service Intelligent (DeSI) Research Lab at the Center for Artificial Intelligence, Faculty of Engineering and Information Technology, University of Technology Sydney, Australia. His research interests include tree similarity measure, recommender systems, and business intelligence.

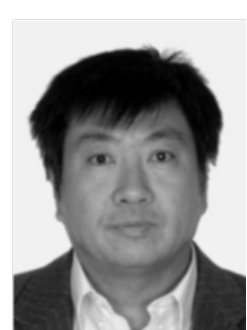

Guangquan Zhang is an Associate Professor and the Director of the Decision Systems and e-Service Intelligent (DeSI) Research Lab at the Center for Artificial Intelligence, Faculty of Engineering and Information Technology, University of Technology Sydney, Australia. He received his $\mathrm{PhD}$ in applied mathematics from Curtin University of Technology, Australia, in 2001. His research interests include fuzzy sets and systems, fuzzy optimization, fuzzy transfer learning, and fuzzy modeling in machine learning and data analytics. He has authored five monographs, five textbooks, and 400 papers including over 200 refereed international journal papers. Dr. Zhang has won seven Australian Research Council (ARC) Discovery Projects grants and many other research grants. $\mathrm{He}$ was awarded an ARC QEII fellowship in 2005. He has served as a member of the editorial boards of several international journals, as a guest editor of eight special issues of IEEE Transactions and other international journals, and has co-chaired several international conferences and workshops in the area of fuzzy decision-making and knowledge engineering. 